\title{
Experiment Design for Complex VTOL Aircraft with Distributed Propulsion and Tilt Wing
}

\author{
Patrick C. Murphy ${ }^{1}$ \\ NASA Langley Research Center, Hampton, VA, 23681 \\ Drew Landman ${ }^{2}$ \\ Old Dominion University, Department of Aerospace Engineering, Norfolk, VA, 23529
}

\begin{abstract}
Selected experimental results from a wind tunnel study of a subscale VTOL concept with distributed propulsion and tilt lifting surfaces are presented. The vehicle complexity and automated test facility were ideal for use with a randomized designed experiment. Design of Experiments and Response Surface Methods were invoked to produce run efficient, statistically rigorous regression models with minimized prediction error. Static tests were conducted at the NASA Langley 12-Foot Low-Speed Tunnel to model all six aerodynamic coefficients over a large flight envelope. This work supports investigations at NASA Langley in developing advanced configurations, simulations, and advanced control systems.
\end{abstract}

\section{Nomenclature}

$\begin{array}{ll}B_{i} & =\text { regression coefficients } \\ C_{N} & =\text { normal force coefficient } \\ C_{m} & =\text { pitching-moment coefficient } \\ \bar{c} & =\text { mean aerodynamic chord, } \mathrm{ft} \\ R^{2} & =\text { multiple correlation coefficent } \\ x_{i} & =\text { regressors }\end{array}$
$\alpha \quad=$ angle-of-attack, deg
$\beta \quad=$ sideslip angle, deg
$\sigma \quad=$ standard error

Abbreviations

ANOVA $=$ Analysis of Variance

BWB = Blended Wing Body

$\mathrm{CCD} \quad=$ Central Composite Design

DEP $=$ Distributed Electric Propulsion

DOE = Design of Experiments

FCD $=$ Face-Centered Design

$\begin{array}{ll}\text { ME } & =\text { Main Effects } \\ \text { OFAT } & =\text { One Factor At a Time } \\ \text { RSM } & =\text { Response Surface Modeling } \\ \text { VIF } & =\text { Variance Inflation Factor } \\ \text { VTOL } & =\text { Vertical Takeoff \& Landing } \\ \text { 2FI } & =\text { Two-factor Interaction }\end{array}$

Aircraft Factors

LTS1 = left tail elevator, deg

aoa = aircraft angle of attack, deg

RTS2 = right tail elevator, deg

beta $=$ aircraft sideslip, deg

LWS1 = left wing outboard surface, deg

Ttilt = horizontal tail position, deg

LWS2 = left wing middle surface, deg

LWS3 = left wing inboard surface, deg

RWS4 = right wing inboard surface, deg

Wtilt = wing position, deg

Rud = rudder position, deg

RWS5 = right wing middle surface, deg

RWS6 = right wing outboard surface, deg

LWEi = left wing engine $\mathrm{i}=1-4$, rpm

RWEj = right wing engine $j=5-8$, rpm

LTE1 = left tail engine, $\mathrm{rpm}$

RTE2 = right tail engine, $r$ m

\footnotetext{
${ }^{1}$ Senior Research Engineer, Dynamic Systems \& Control Branch, MS 308, Associate Fellow.

2 Professor, Department of Aerospace Engineering, 3750 Elkhorn Avenue, Associate Fellow.
} 


\section{Introduction}

$\mathrm{T}$ The science behind Design of Experiments (DOE) has been developing since the 1800s with early work such as "Theory of Probable Inference," by Charles S. Pierce, and later with work by Ronald A. Fisher, "The Design of Experiments ${ }^{1}$," that effectively established the field of DOE. The field has since expanded and modernized from Fisher's agricultural applications to all fields of modern science and engineering.

Surprisingly, application of DOE theory is not wide spread in aerospace literature, particularly in application to wind tunnel testing and modeling aerospace vehicles. This likely reflects the fact that many existing wind tunnel facilities were not originally designed to accommodate DOE testing methods. Some example applications are investigations by DeLoach ${ }^{2-4}$, considering various aspects of DOE applied to wind tunnel testing. Examples with more complex aircraft have been made by Landman $^{5-6}$. Reference 5 describes application of Response Surface Modeling (a subset of DOE) to the Boeing Blended Wing Body (BWB) configuration. In the BWB case some surfaces were ganged together reducing the total number of factors to 8 . An efficient fractional factorial design, augmented to a facecentered design (FCD), was created to capture main effects, two and three factor interactions, and pure quadratic terms. In Ref. 6 a novel nested FCD was created for the X-31 aircraft with 5 factors. This design incorporated the advantages of a fractional FCD with 5-levels of measurements. The experiment design ensured 5 equal levels for control surface deflections that cover the full range of motion. Capturing the full range of motion is an important aspect of modeling aircraft control effectors.

This paper highlights an experimental wind tunnel investigation where DOE theory ${ }^{7}$ is applied to obtain static aerodynamic models for an advanced and complex aircraft concept. The concept vehicle GL-10 was developed at NASA Langley Research Center. The vehicle is an unmanned aircraft shown in Fig. 1. GL-10 is a VTOL aircraft designed with a distributed electric propulsion system, tilt wing, tilt tail, and nine control surfaces (six flaperons, two elevators, one rudder). Details of the GL-10 design and development are described in Refs. 8-10. The design capitalizes on a distributed electric propulsion system that allows thrust to be applied across the airframe without mechanical complexity and allows a broad scale-free range for the designer. Electric propulsion opens the design space in terms of efficiency and metrics such as power-to-weight ratio, especially for VTOL aircraft. In hover mode both the wing and horizontal tail rotate to a vertical position with all ten propellers working. Eight propellers are stationed across the wing and two on the horizontal tail. In cruise mode the aircraft operates as a normal aircraft with only the two outboard propellers operating on the wings and the remaining props folded.

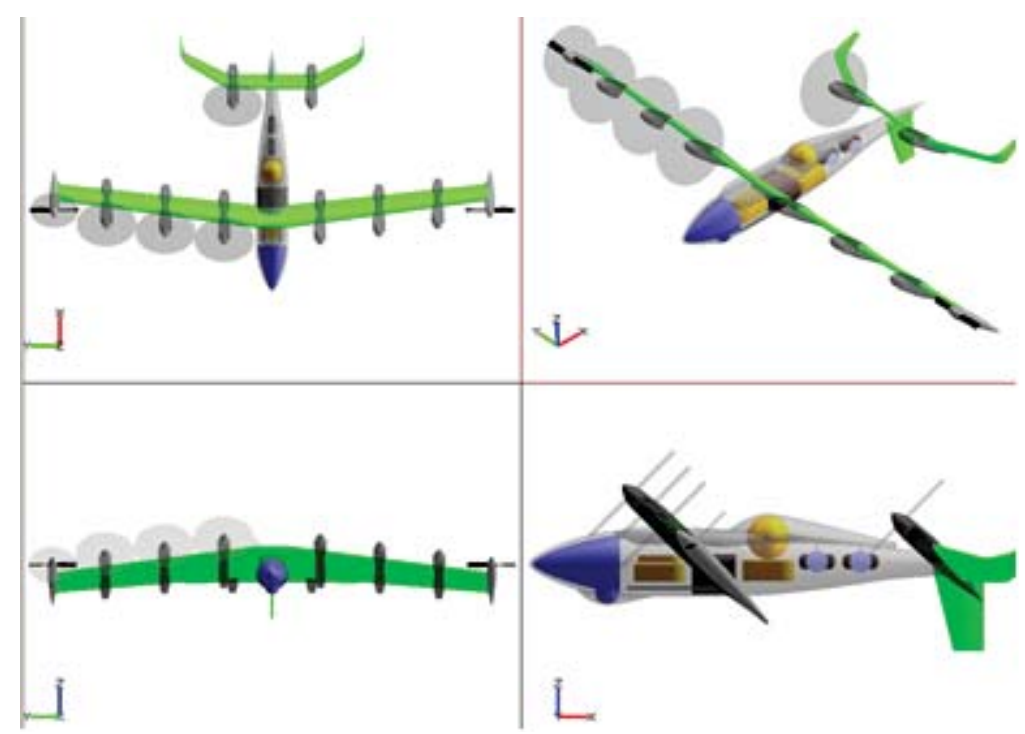

Fig. 1 GL-10 images showing various configurations of flight.

Two requirements for GL-10 model development were to characterize static aerodynamics in a relatively short period of time and at a relatively low cost. A project decision determined that somewhat larger confidence intervals could be tolerated in order to save time and reduce costs. The GL-10 all-electric prototype control system commands 21 control variables, ten of which are propulsion commands. Two of the control signals, wing tilt and tail tilt,

American Institute of Aeronautics and Astronautics 
significantly change the vehicle's geometric configuration and move the multi-body center of gravity. Nine control signals move three surfaces on each wing, one surface on each horizontal tail, and one surface on a vertical ventral tail. The GL-10's flight envelope encompasses a large range of vehicle configurations, airspeeds, and flight attitudes. Including angle of attack and sideslip, a total of 23 variables or factors required investigation in order to properly model this vehicle.

Characterizing GL-10 aerodynamics under these requirements presents a number of challenges to conventional methods of test where one factor at a time (OFAT) is varied while holding all other variables constant. The first challenge is that the test time required is prohibitive due to the large number of factors involved. A test matrix to map 23 factors and their interactions would take years of conventional testing. The second challenge is handling system errors. The conventional OFAT method assumes testing apparatus remain stable during the testing period. Although corrections for temperature and zero drift in measurement systems are frequently checked, it becomes more difficult in longer test runs. Also the OFAT approach inherently captures any unknown systematic errors. Unfortunately, any errors due to the testing system become confounded with the precision errors and cannot be separated. A third challenge is that the conventional approach does not readily lend itself to detecting response changes due to interactions between input variables. Obtaining cross products (interactions) in the response polynomial models require input designs that produce responses with the proper information content to allow identification of these and other higher order terms. As Fig. 2 suggests, a number of technical disciplines address the issue of modeling in the presence of uncertainty, however, the least desirable approach is the conventional OFAT method. The blind researchers investigating the elephant in Fig. 2 reflect the reality captured in a quote by Box ${ }^{11}$, "Essentially, all models are wrong, but some are useful."

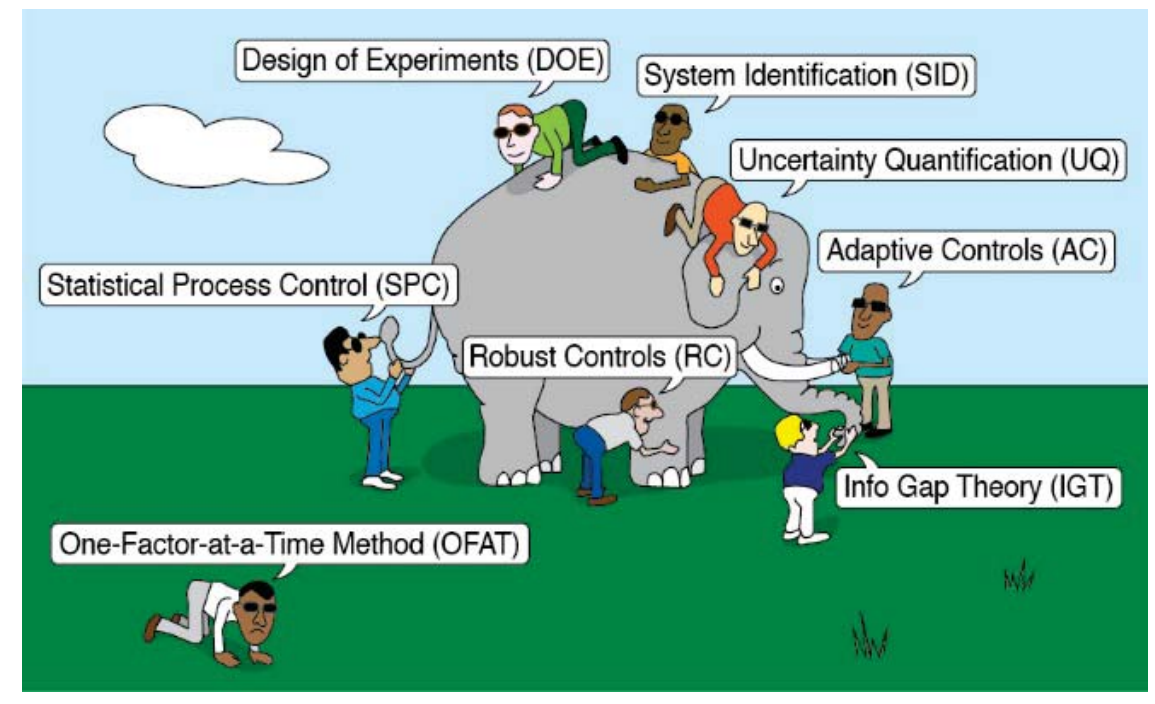

Fig. 2 Uncertainty Viewpoints

\section{DOE Applied to GL-10}

The solution to GL-10 modeling challenges was a series of experiments (including up to a 23 factor experiment) designed using Design of Experiments or specifically Response Surface Modeling (RSM) theory ${ }^{7}$ that produced extremely efficient, statistically rigorous designs that minimized prediction error. DOE theory gives researchers a methodology for defining experiments that are very efficient in estimating the effects of input variables, their interactions, and sensitivities of the responses to these inputs. This is done in a statistically rigorous framework that allows optimizations of the designs to produce more robustness with respect to various types of error. In addition, the experimenter can a priori determine how much statistical power is available for the design and ensure that sufficient data is collected to achieve the desired statistical performance. The testing was also facilitated by specific mechanical automation and software capabilities provided in Langley's 12-Foot Wind Tunnel discussed in Ref. 8.

\section{A. Supporting Basic Tenets of DOE}

Facility capabilities described in Ref. 8 allowed the basic principles of DOE to be readily incorporated into the test. Four key principles in DOE theory have a direct bearing on the wind tunnel setup and operational requirements: 
1. Orthogonal regressors - orthogonal regressors are uncorrelated to improve estimation calculations.

2. Replication - independent and repeated measurements to assess system noise and uncertainty.

3. Randomization - randomized input test matrix to average out extraneous factors and systematic errors.

4. Blocking - technique to improve precision and reduce variability due to known nuisance factors.

Effectively these principles place requirements on the test facility that can be accommodated by three capabilities: automation, speed, and accurate arbitrary motion. Automation addresses replication and randomization by providing the abilities to command any position of the model, test rig, and all model actuators from a pre-defined user test matrix, followed by recording of input commands, achieved set points (verified by measurements), and aerodynamic responses. Ideally feedback control should be used to ensure set points are achieved as commanded. This is important for repeatability and ensuring regressors achieve their orthogonal design characteristics. Ideally all this information is collected as fast and accurately as possible and then transmitted back in the exact sequence tested to the investigators for analysis. Rapid data acquisition is a key factor that allows a very large number of test points to be collected in a short time. Precision is also helped by incorporating blocking into the experiment design to remove the effects of known sources of error. Blocking to limit the length of each test was also essential for this particular facility since tests longer than a few hours would cause significant overheating of the tunnel motors and tunnel environment.

\section{B. Test Design: The I-Augmented, Nested, Face Centered Design}

The foundation for DOE classical designs is the factorial experiment ${ }^{1}$. A factorial design is one which varies all factors simultaneously for all possible combinations. Using two levels for each factor represents a run-efficient method for developing a first order plus interaction model. A full-factorial design in two factor space is shown below in Fig. 3 with the addition of a point at the origin known as a center.

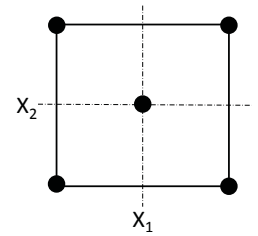

Figure 3. A full Factorial design in two-factor space.

Replicated centers afford a test for possible augmentation to support quadratic model terms. The supported regression model is shown with up to two-factor interactions (2FI) by

$$
y=B_{0}+\sum_{i} B_{i} x_{i}+\sum \sum_{i \neq j} B_{i j} x_{i} x_{j}+\ldots+\varepsilon \quad i=1,2, \ldots, k
$$

The B's are the fitted regression coefficients and the $x$ 's are the factors (independent variables). A refinement (augmentation) to the factorial design is the central composite design ${ }^{12}$ (CCD) which adds design points along the axes through the origin of the design space as shown by the square symbols of Fig. 4.

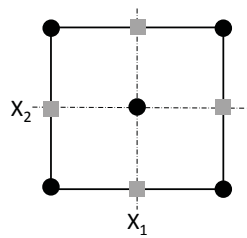

Figure 4. A face-centered central composite design in two-factor space. 
This approach supports a full second order model given in Eq. (2).

$$
y=B_{0}+\sum_{i} B_{i} x_{i}+\sum_{i} B_{i i} x_{i}^{2}+\sum_{i \neq j} B_{i j} x_{i} x_{j}+\varepsilon \quad i=1,2, \ldots, k
$$

The location of the axial points defines this CCD as a face-centered design (FCD). The nested face-centered design is a recent development which allows the nesting of two FCD designs to support the addition of pure cubic terms to the empirical model. The two FCD's may be tuned by fractionating the factorial designs as presented by Landman et al. in Ref. 6. The design in two-factor space is shown in Fig. 5.

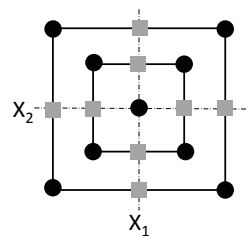

Figure 5. A nested face-centered design in two-factor space.

where the supported model is given by Eq. (3).

$$
y=B_{0}+\sum_{i} B_{i} x_{i}+\sum_{i} B_{i i} x_{i}^{2}+\sum_{i \neq j} B_{i j} x_{i} x_{j}+\sum_{i} B_{i i i} x_{i}^{3}+\varepsilon \quad i=1,2, \ldots, k
$$

Initial designs in this study for GL-10 were attempted using the nested FCD concept which has been described in detail for use with five factors in Ref. 6. In that study both inner nested FCD and outer FCD were half-fraction factorial designs producing an efficient 5-level, 55-run test with three center points. Simple dimensional expansion beyond 5 factors yields a design which suffers from lack of orthogonality and an increase in parameter variance which quickly degrades as the number of factors are increased. Correlation in the parameter estimates was monitored in the design process using the variance inflation factor (VIF) metric. The VIF represents a measure of multicolinearity or simply stated, a measure of the ability to obtain unique estimates of the regression coefficients. Low values of VIF are desired where a perfectly unique estimate occurs with a VIF of one. A rule-of-thumb used by DOE practitioners ${ }^{13}$ is to try to keep VIF values below 10. A solution was found by blending the nested-FCD design points with additional design points found using an I-optimal algorithm. An I-optimal design is one based on minimizing the integrated prediction variance over the design space ${ }^{7}$. The statistical power to estimate regression model terms was evaluated for a difference-to-detect of two standard deviations in the response using a 5\% level of significance. The I-optimal augmentation proved beneficial in that the benefits of the nested-FCD were retained, model identification was enhanced, and only a relatively moderate number of test points were added. Validation points were chosen using the same I-optimal algorithm so that they could be used to augment the model or to serve as independent confirmation points.

Design metrics for the designs used are shown in Tables 1-3. Maximum values of the design metrics show how each design was developed and improved as blocks of additional design points were added to make the final designs. Table 1 shows results for Cruise and Loiter modes where 14 factors were considered, Table 2 shows the Transition mode with 23 factors, and Table 3 presents the Hover mode with 19 factors. Cruise and Loiter mode tests used the same design but differed in the range of angle of attack. Loiter mode flight operations are designed for flight close to stall and thus is a more nonlinear aerodynamic region. The number of runs in the first block of each design is a direct result of the number of factors applied to a minimum-run, resolution V, FCD design with center points. Resolution is a measure that reflects the degree to which regressors are confounded or aliased. Resolution $\mathrm{V}$ reflects designs in which no main effect (ME) or two-factor interaction (2FI) is aliased with any other main effect or two-factor iteraction ${ }^{13}$. The second block, forming the nested FCD, is a replicate block scaled for nesting. The basic designs were built around a full quadratic model structure, however, sufficient degrees of freedom were available for cubic terms. For the transition mode, with 23 factors, the first block required 330 runs. This number of runs was also a comfortable limit that avoided tunnel overheating. Consequently, blocks 1 and 2 were run separately for the transition and hover modes. All remaining blocks were run in sequence and the order of runs within a given block was fully randomized.

American Institute of Aeronautics and Astronautics 
Table 1. Maximum Values of Design Metrics for Cruise and Loiter Modes (14 Factors)

\begin{tabular}{|l|l|c|c|c|c|}
\hline Block Type & Blocks & Runs & Terms & VIF & $\%$ Power \\
\hline & included & & & & $2 \sigma, \mathrm{s} / \mathrm{n}=2$ \\
\hline & & & & & \\
\hline FCD Min Res V & 1 & 140 & ME & 3.37 & 99.9 \\
\hline FCD Min Res V & 1 & 140 & 2FI & 3.85 & 99.9 \\
\hline FCD Min Res V & 1 & 140 & Quadratic & 11.47 & 79.7 \\
\hline & & & & & \\
\hline Nested FCD & 1,2 & 280 & ME & 3.02 & 99.9 \\
\hline Nested FCD & 1,2 & 280 & 2FI & 3.77 & 99.9 \\
\hline Nested FCD & 1,2 & 280 & Quadratic & 21.71 & 85.2 \\
\hline & & & & & \\
\hline I-optimal & $1,2,3$ & 350 & ME & 1.43 & 99.9 \\
\hline I-optimal & $1,2,3$ & 350 & 2FI & 1.74 & 99.9 \\
\hline I-optimal & $1,2,3$ & 350 & Quadratic & 5.03 & 99.9 \\
\hline & & & & & \\
\hline Validation (I-opt) & $1, \ldots, 4$ & 385 & ME & 1.28 & 99.9 \\
\hline Validation (I-opt) & $1, \ldots, 4$ & 385 & 2FI & 1.48 & 99.9 \\
\hline Validation (I-opt) & $1, \ldots, 4$ & 385 & Quadratic & 3.69 & 99.9 \\
\hline & & & & & \\
\hline Validation (I-opt) & $1, \ldots, 4$ & 385 & *Pure Cubic & 13.06 & 95.9 \\
\hline
\end{tabular}

*Pure Cubic terms added to quadratic model

Table 2. Maximum Values of Design Metrics for Transition Modes (23 Factors)

\begin{tabular}{|l|l|c|c|c|c|}
\hline Block Type & Blocks & Runs & Terms & VIF & \% Power \\
\hline & included & & & & $2 \sigma, \mathrm{s} / \mathrm{n}=2$ \\
\hline & & & & & \\
\hline FCD Min Res V & 1 & 330 & ME & 4.30 & 99.9 \\
\hline FCD Min Res V & 1 & 330 & 2FI & 5.35 & 99.9 \\
\hline FCD Min Res V & 1 & 330 & Quadratic & 20.29 & 79.9 \\
\hline & & & & & \\
\hline Nested FCD & 1,2 & 660 & ME & 3.63 & 99.9 \\
\hline Nested FCD & 1,2 & 660 & 2FI & 5.07 & 99.9 \\
\hline Nested FCD & 1,2 & 660 & Quadratic & 50.37 & 84.4 \\
\hline & & & & & \\
\hline I-optimal & $1,2,3$ & 775 & ME & 1.88 & 99.9 \\
\hline I-optimal & $1,2,3$ & 775 & 2FI & 2.20 & 99.9 \\
\hline I-optimal & $1,2,3$ & 775 & Quadratic & 7.05 & 99.9 \\
\hline & & & & & \\
\hline Validation (I-opt) & $1, \ldots, 4$ & 850 & ME & 1.54 & 99.9 \\
\hline Validation (I-opt) & $1, \ldots, 4$ & 850 & 2FI & 1.80 & 99.9 \\
\hline Validation (I-opt) & $1, \ldots, 4$ & 850 & Quadratic & 4.92 & 99.9 \\
\hline & & & & & \\
\hline Validation (I-opt) & $1, \ldots, 4$ & 850 & *Pure Cubic & 12.07 & 99.9 \\
\hline
\end{tabular}

*Pure Cubic terms added to quadratic model

The tables demonstrate VIF levels that are too high (greater than 10) when a relatively large number of factors are applied to a nested FCD, represented by blocks 1 and 2. The high VIF levels are reduced by blending in I-optimal design points as shown when block 3 is included. Retaining the underlying nested FCD allowed the full range of actuation to be tested with 5 levels (an important feature for aircraft applications) and blending optimized points provided acceptable levels for VIF as well as producing a design that minimized prediction error. No constraints were required for any of the factors in this study and all the factors were "easy to change" avoiding the need for split-plot 
designs. The number of optimal points selected was chosen to be 5 times the number of factors. This was generally found to provide an acceptable VIF as shown in Tables 1-3 for each of the 3-block design cases. A fourth block was added to the design to provide validation points. Models presented in this paper utilize runs from all four blocks. For the fourth block, the number of runs chosen was approximately $10 \%$ of the total runs in blocks 1-3.

Table 3. Maximum Values of Design Metrics for Hover Mode (19 Factors)

\begin{tabular}{|l|l|c|c|c|c|}
\hline Block Type & Blocks & Runs & Terms & VIF & \% Power \\
\hline & included & & & & $2 \sigma, \mathrm{s} / \mathrm{n}=2$ \\
\hline & & & & & \\
\hline FCD Min Res V & 1 & 236 & ME & 3.99 & 99.9 \\
\hline FCD Min Res V & 1 & 236 & 2FI & 4.50 & 99.9 \\
\hline FCD Min Res V & 1 & 236 & Quadratic & 16.36 & 79.9 \\
\hline & & & & & \\
\hline Nested FCD & 1,2 & 472 & ME & 3.40 & 99.9 \\
\hline Nested FCD & 1,2 & 472 & 2FI & 4.35 & 99.9 \\
\hline Nested FCD & 1,2 & 472 & Quadratic & 36.36 & 84.7 \\
\hline & & & & & \\
\hline I-optimal & $1,2,3$ & 567 & ME & 1.67 & 99.9 \\
\hline I-optimal & $1,2,3$ & 567 & 2FI & 1.89 & 99.9 \\
\hline I-optimal & $1,2,3$ & 567 & Quadratic & 6.19 & 99.9 \\
\hline & & & & & \\
\hline Validation (I-opt) & $1, \ldots, 4$ & 622 & ME & 1.46 & 99.9 \\
\hline Validation (I-opt) & $1, \ldots, 4$ & 622 & 2FI & 1.64 & 99.9 \\
\hline Validation (I-opt) & $1, \ldots, 4$ & 622 & Quadratic & 4.67 & 99.9 \\
\hline & & & & & \\
\hline Validation (I-opt) & $1, \ldots, 4$ & 622 & *Pure Cubic & 12.36 & 99.8 \\
\hline
\end{tabular}

*Pure Cubic terms added to quadratic model

\section{Aerodynamic Modeling Experiments for GL-10}

Respecting the project demand for low-cost and rapid modeling of GL-10 aerodynamics forced the authors toward innovative experiment designs. A complex aircraft with 23 factors does not lend itself to a conventional experiment without significantly limiting the number of factors and size of the flight envelope investigated or accepting an extremely lengthy test program. To avoid these limitations and to capture a variety of different test objectives, experiments were developed using DOE/RSM techniques to satisfy the project goals.

\section{A. Test Objectives}

The first experiments were exploratory, designed mostly as conventional tests, to help establish some general characteristics of the aircraft and evaluate certain features. These tests define appropriate subspace centers, model order, ranges for the major aerodynamic factors, control effectors, and provide some conventional data to aid in evaluating test results. Data sweeps were done to determine the presence and severity of any static hysteresis. A test to assess if the aircraft benefited from using vortilons was also performed. Since total test-time was an important parameter to minimize, a designed experiment was developed to evaluate the data acquisition system sample times and determine how much it could be shortened, if at all. The standard sample time used in the facility was 10 seconds. In wind tunnel environments vibrations, flow turbulence, test rig motions and vibrations, etc., all add a relatively large amount of noise to the measurements so taking an average measurement is common practice. Based on years of testing, 10 seconds was determined to provide very robust and repeatable results. Screening tests were primarily done with props off due to the limited time available.

The second group of experiments was composed of five sets of DOE/RSM-based experiments. Each of the five sets was uniquely designed to model one of the four modes of flight: cruise, loiter, transition, and hover. The transition mode was split into a low-transition mode where the wing tilt ranged from $0^{\circ}$ to $45^{\circ}$ and a high-transition mode where the wing tilt ranged from $45^{\circ}$ to $90^{\circ}$. The primary objective for the experiment was aerodynamic characterization of the GL-10 for all modes of flight. Experiments were designed for quadratic models and cubic terms, if present. In

American Institute of Aeronautics and Astronautics 
general 3-factor interactions are aliased in such designs but the optimized design had substantial degrees-of-freedom that allowed some limited 3-factor terms and typical cubic terms with respect to alpha and beta to be uniquely identified. These objectives included obtaining a 95\% confidence level on parameter estimates and a minimum $80 \%$ level for statistical power. The distributed electric propulsion (DEP) system enhances propulsion-aerodynamic interactions, however, from a modeling perspective this enhancement is a significant added complication given the potential for numerous complex interactions.

\section{B. Facility and Model Details}

Testing was performed in the NASA Langley 12-Foot Low Speed Wind Tunnel. Although only static testing was performed in this study, the facility is capable of both static and dynamic testing as well as a variety of flow visualization techniques. A side view of this test facility is shown in Fig. 6. It is a closed-throat, atmospheric tunnel with an annular return. The test section is 8 sided, 12 feet wide, and 15 feet long. Angle of attack range is from $-10^{\circ}$ to $90^{\circ}$, sideslip range is $-90^{\circ}$ to $90^{\circ}$, and velocity can be varied from 0 to $77 \mathrm{fps}$. More details of the facility are provided in Ref. 8.

A key feature of this facility is that both hardware and tunnel control software have been adapted to allow DOEbased testing in addition to conventional OFAT sweeps. The facility was modified to minimize mechanical slop and positioning errors and software was modified to allow pre-programmed, automated, and randomized movement through a DOE test matrix. The data acquisition system records and returns all commanded independent parameters and output measurements in the sequence requested and in a single output file. Force and moment measurements for the GL-10 test included data from a 6-component strain gauge balance inside the model. These data were averaged over a sampling period determined in an exploratory test discussed in the next section.

A significant change to tunnel software was also required to accommodate weight tare effects. Configuration changes that occur when the wing and tail are moved over a range of tilt angles result in longitudinal and vertical position changes of the center of gravity and thus weight tare effects are not constant during any DOE run. As a practical matter for this study, tares were measured at each test point for each complete block of runs. The tare runs were made with tunnel wind off and with motors off while recording forces and moments due to gravity. All independent factors, except for the motors, were set to their test point values during the tare run. To characterize the total force and moment contributions of the combined aerodynamic effects and propulsion effects, measurements were taken with wind on and propulsion on. The tunnel data acquisition and processing system applied tare values to the corresponding data points obtained in subsequent DOE runs with wind on and motors running. Additional model characterization was made with just propulsion systems on and wind off. Finally, a single engine without the aircraft body was investigated to characterize pure propeller effects.

The GL-10 wind tunnel model is a $56.25 \%$ scale model of a sub-scale flight test vehicle. Model wingspan is 70.2 inches, wing area is 361.2 square inches, and mean chord is 5.44 inches. The model represents a unique VTOL design with 10 propellers and 11 surface controls. Depending on the flight mode tested, a total of up to 23 factors were required. Tables 4-6 show the factors and factor ranges for each mode tested.

Table 4 summarizes factors and factor ranges for the Cruise and Loiter modes. The primary difference between these two modes is in the range of angle of attack. Cruise mode was designed for normal aircraft flight at relatively low angles of attack from $-5^{\circ}$ to $10^{\circ}$. Loiter mode reflects the aircraft designer's intent for lower speed, high-lift flight near stall conditions. Consequently, the experiment for loiter mode was designed for a higher range of angle of attack where a more nonlinear aerodynamic response occurs. The loiter mode was tested in an alpha range covering $5^{\circ}$ to $20^{\circ}$. Idle speed to maximum speed for the motors was specified to be $4000 \mathrm{rpm}$ and $12000 \mathrm{rpm}$, respectively.

Table 5 summarizes factors for the Transition mode. The main feature of this mode is the transition of the wing from a normal cruise position to a vertical $90^{\circ}$ hover-mode position. The transition mode was tested in two wing-tilt ranges because the wing-tilt actuator was only capable moving the wing through $45^{\circ}$. The wing tilt position is measured from the stowed position $\left(0^{\circ}\right.$ tilt) which places the wing at approximately $4^{\circ}$ higher incidence than the fuselage water-line. Consequently the wing experiences approximately $4^{\circ}$ higher alpha than the aircraft alpha. The low transition design used a wing-tilt factor range of $0^{\circ}$ to $45^{\circ}$ and the high transition design used $45^{\circ}$ to $90^{\circ}$. Aircraft angle of attack was limited between 0 and 15 since the aircraft fuselage would not likely require very high angles of attack during transition. The expectation for this aircraft was that as the transition occurred from cruise to hover that the aircraft would substantially slow down with the fuselage remaining in relatively low pitch attitude and lower alpha while the wing would rapidly see higher angles of attack until reaching a 90 orientation to the fuselage.

American Institute of Aeronautics and Astronautics 


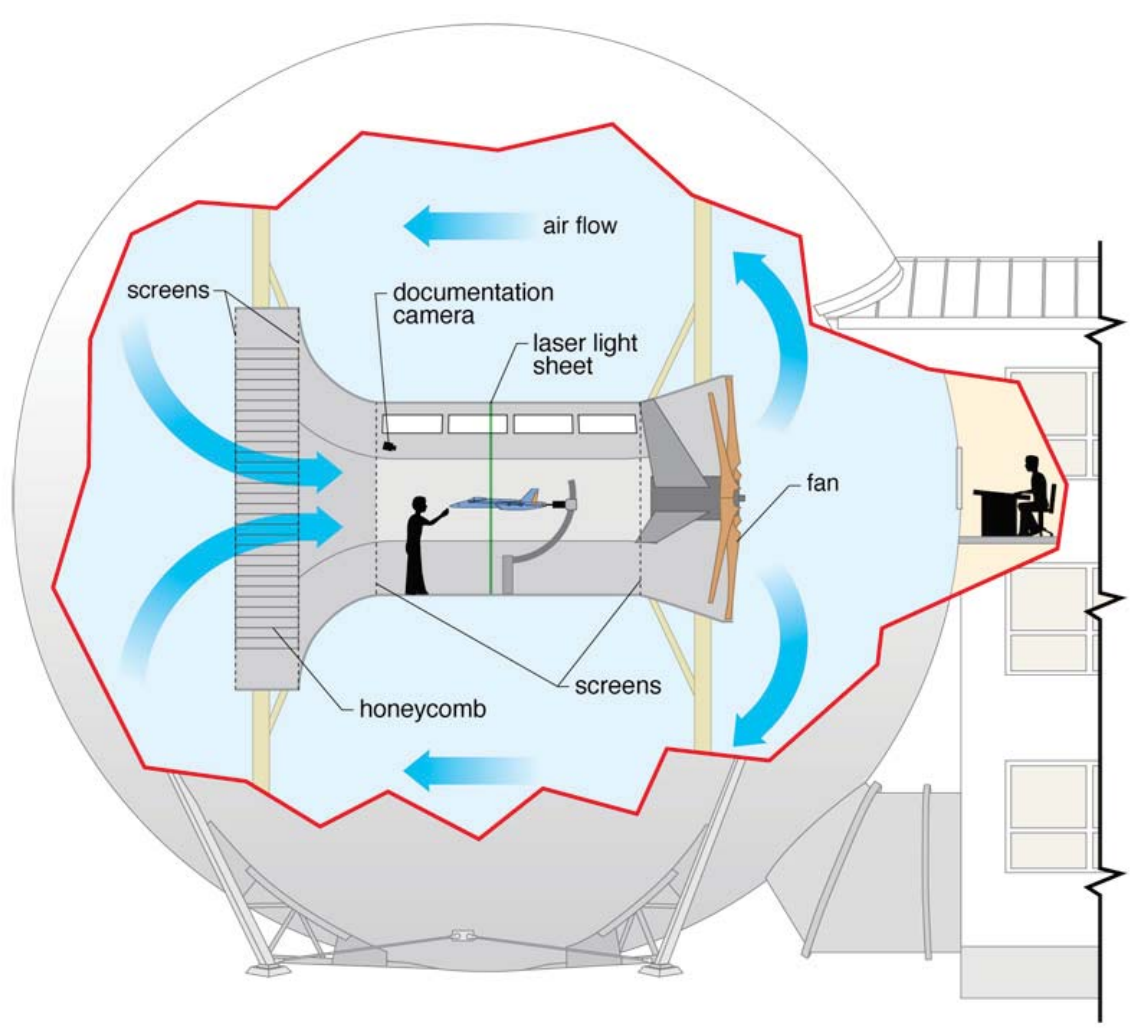

Fig. 6 NASA Langely 12-Foot Low Speed Tunnel

Table 4. Factor Ranges for Cruise and Loiter Modes

\begin{tabular}{|l|c|l|l|l|l|l|c|}
\hline No. & Term & Label & Description & Low & High & Units & Notes \\
\hline & & & & Range & Range & & \\
\hline 1 & A & aoa & Aircraft alpha & -5 & 20 & deg & [-5:10] Cruise; [5:20] Loiter \\
\hline 2 & B & beta & Aircraft beta & -5 & 5 & deg & \\
\hline 3 & C & LWS1 & Surface 1 left wing & -40 & 25 & deg & TED = positive \\
\hline 4 & D & LWS2 & Surface 2 left wing & -40 & 25 & deg & “ \\
\hline 5 & E & LWS3 & Surface 3 left wing & -40 & 25 & deg & “ \\
\hline 6 & F & RWS4 & Surface 4 right wing & -40 & 25 & deg & “ \\
\hline 7 & G & RWS5 & Surface 5 right wing & -40 & 25 & deg & “ \\
\hline 8 & H & RWS6 & Surface 6 right wing & -40 & 25 & deg & “ \\
\hline 9 & J & LTS1 & Surface 7 left tail & -40 & 25 & deg & “ \\
\hline 10 & K & RTS2 & Surface 8 right tail & -40 & 25 & deg & TER = positive \\
\hline 11 & L & Ttilt & Tail tilt & -5 & 5 & deg & deg \\
\hline 12 & M & Rud & Rudder & -25 & 25 & deg & Th \\
\hline 13 & N & LWE1 & Left Wing Eng 1 & 4000 & 12000 & rpm & \\
\hline 14 & O & RWE8 & Right Wing Eng 8 & 4000 & 12000 & rpm & \\
\hline
\end{tabular}


Table 5. Factor Ranges for Transition Mode

\begin{tabular}{|l|c|l|c|l|l|l|c|}
\hline No. & Term & Label & Description & Low & High & Units & Notes \\
\hline & & & & Range & Range & & \\
\hline 1 & A & aoa & Aircraft alpha & 0 & 15 & deg & \\
\hline 2 & B & beta & Aircraft beta & -5 & 5 & deg & \\
\hline 3 & C & LWS1 & Surface 1 left wing & -40 & 25 & deg & TED = positive \\
\hline 4 & D & LWS2 & Surface 2 left wing & -40 & 25 & deg & “ \\
\hline 5 & E & LWS3 & Surface 3 left wing & -40 & 25 & deg & “ \\
\hline 6 & F & RWS4 & Surface 4 right wing & -40 & 25 & deg & “ \\
\hline 7 & G & RWS5 & Surface 5 right wing & -40 & 25 & deg & “ \\
\hline 8 & H & RWS6 & Surface 6 right wing & -40 & 25 & deg & “ \\
\hline 9 & J & LTS1 & Surface 7 left tail & -40 & 25 & deg & “ \\
\hline 10 & K & RTS2 & Surface 8 right tail & -40 & 25 & deg & \\
\hline 11 & L & Wtilt & Wing tilt & 0 & 90 & deg & [0:45] low; [45:90] high \\
\hline 12 & M & Ttilt & Tail tilt & -8 & 98 & deg & [-8:50] low; [45:98] high \\
\hline 13 & N & Rud & Rudder & -25 & 25 & deg & TER = positive \\
\hline 14 & O & LWE1 & Left Wing Eng 1 & 4000 & 12000 & rpm & \\
\hline 15 & P & LWE2 & Left Wing Eng 2 & 4000 & 12000 & rpm & \\
\hline 16 & Q & LWE3 & Left Wing Eng 3 & 4000 & 12000 & rpm & \\
\hline 17 & R & LWE4 & Left Wing Eng 4 & 4000 & 12000 & rpm & \\
\hline 18 & S & RWE5 & Right Wing Eng 5 & 4000 & 12000 & rpm & \\
\hline 19 & T & RWE6 & Right Wing Eng 6 & 4000 & 12000 & rpm & \\
\hline 20 & U & RWE7 & Right Wing Eng 7 & 4000 & 12000 & rpm & \\
\hline 21 & V & RWE8 & Right Wing Eng 8 & 4000 & 12000 & rpm & \\
\hline 22 & W & LTE1 & Left Tail Eng 1 & 4000 & 12000 & rpm & \\
\hline 23 & X & RTE2 & Right Tail Eng 2 & 4000 & 12000 & rpm & \\
\hline
\end{tabular}

Table 6. Factor Ranges for Hover Mode

\begin{tabular}{|l|c|l|l|l|l|l|l|}
\hline No. & Term & Label & Description & Low & High & Units & Notes \\
\hline & & & & Range & Range & & \\
\hline 1 & A & LWS1 & Surface 1 left wing & -40 & 25 & deg & TED = positive \\
\hline 2 & B & LWS2 & Surface 2 left wing & -40 & 25 & deg & “ \\
\hline 3 & C & LWS3 & Surface 3 left wing & -40 & 25 & deg & “ \\
\hline 4 & D & RWS4 & Surface 4 right wing & -40 & 25 & deg & “ \\
\hline 5 & E & RWS5 & Surface 5 right wing & -40 & 25 & deg & “ \\
\hline 6 & F & RWS6 & Surface 6 right wing & -40 & 25 & deg & “ \\
\hline 7 & G & LTS1 & Surface 7 left tail & -40 & 25 & deg & positive \\
\hline 8 & H & RTS2 & Surface 8 right tail & -40 & 25 & deg & \\
\hline 9 & J & Rud & Rudder & -25 & 25 & deg & TER = \\
\hline 10 & K & LWE1 & Left Wing Eng 1 & 4000 & 12000 & rpm & \\
\hline 11 & L & LWE2 & Left Wing Eng 2 & 4000 & 12000 & rpm & \\
\hline 12 & M & LWE3 & Left Wing Eng 3 & 4000 & 12000 & rpm & \\
\hline 13 & N & LWE4 & Left Wing Eng 4 & 4000 & 12000 & rpm & \\
\hline 14 & O & RWE5 & Right Wing Eng 5 & 4000 & 12000 & rpm & \\
\hline 15 & P & RWE6 & Right Wing Eng 6 & 4000 & 12000 & rpm & \\
\hline 16 & Q & RWE7 & Right Wing Eng 7 & 4000 & 12000 & rpm & \\
\hline 17 & R & RWE8 & Right Wing Eng 8 & 4000 & 12000 & rpm & \\
\hline 18 & S & LTE1 & Left Tail Eng 1 & 4000 & 12000 & rpm & \\
\hline 19 & T & RTE2 & Right Tail Eng 2 & 4000 & 12000 & rpm & \\
\hline
\end{tabular}


Table 6 summarizes the 19 factors for Hover mode. In this mode the wing and tail are both locked vertically at $90^{\circ}$ and tunnel velocity is set to zero. This setup represents the hover flight condition where without forward speed the aircraft alpha and beta are not defined. Limited resources prevented testing of various forward speeds for this and other modes.

\section{Exploratory Experiments}

Exploratory experiments were performed using both DOE and conventional test methods to observe aerodynamic behaviors and resolve several key issues. Key issues addressed in advance of the main DOE test were to determine minimum duration of time to sample data or "dwell time" and to assess the degree of static hysteresis present. As part of the exercise to look at hysteresis, the data also allowed observation and confirmation of expected aerodynamic nonlinearities. For this study, the range of sideslip was limited to a benign region of $\pm 5^{\circ}$. This reflects the designer's expectation for controlled flight throughout all flight modes.

Other tests included a short test to determine if vortilons would improve lift during loiter. A vortilon is a flat plate attached to the underside of a wing designed to improve lift by generating a vortex from span wise flow that energizes the boundary layer over the wing. These tests demonstrated the vortilons to be ineffective. Designers believe the four engine pylons on each wing provided the same effect. Additional tests were performed as part of the preliminary testing that included checking for flutter, excessive vibrations, and balance sensitivity over a range of dynamic pressures. A dynamic pressure of $3.2 \mathrm{psf}$ was chosen for the overall test.

\section{Dwell Time Test}

In light of the model complexity, numerous data points required, and limited test time available, a key preliminary step in this study was to find the minimum time required to obtain each data point without sacrificing statistical significance. Although in prior tests a range of data sampling strategies have been used, the default practice for static testing in this facility was to sample at $100 \mathrm{~Hz}$, dwell at each test point for 10 seconds, and use the average value. An exploratory experiment using a nested FCD considered dwell times of 2, 4, 6, 8, and 10 seconds was designed to determine the minimum dwell time with adequate accuracy. The objective for this test was to evaluate dwell times over a wide range of test conditions and not to find a detailed model of the aerodynamic functions. Factors chosen included sideslip, angle of attack and dwell time. The nested FCD design space is illustrated in Fig. 7 using two of the three factors, dwell time and angle of attack. A similar chart exists for each factor pair. The figure shows the design construction as two blocks separating the inner and outer FCD. Aerodynamic force and moment measurements were obtained for the range of dwell times and angles of attack shown and over a broad range sideslip from $-15^{\circ}$ to $+15^{\circ}$.

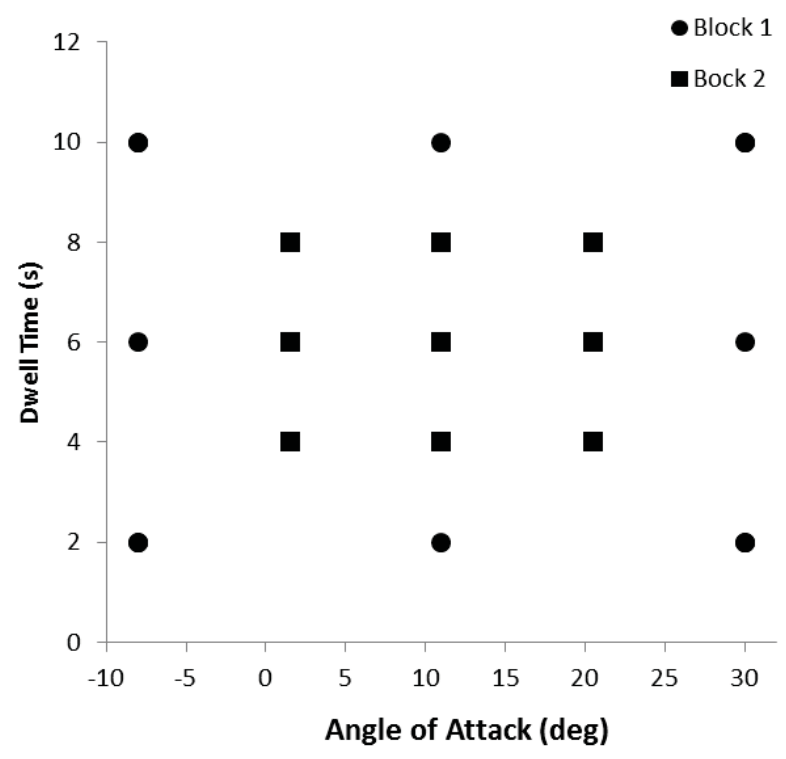

Figure 7. Dwell Test Nested FCD Design in Two-Factor Space

Using ANOVA, dwell time and its interactions were found to be insignificant at the 95\% confidence level. These results set the sample times to two seconds for all subsequent experiments. This initial experiment provided an

American Institute of Aeronautics and Astronautics 
assessment of overall uncertainty due to the model/wind tunnel systems. Sample results of Fig. 8 show the normal force coefficient plotted versus angle of attack with the $95 \%$ confidence bands displayed as dashed lines. Sideslip is set to zero and overlapping measured points (red dots) reflect the small change in measurements with dwell time. The ANOVA for normal force is summarized in table 7 and shows that dwell time is not a significant factor, a result found for all aerodynamic coefficients. The ANOVA also shows the block effect is non-trivial. Further experimentation would need to include blocks to guard against unknown sources of variance. Residual diagnostics were consulted and found to support assumptions of normality, independence and constant variance.

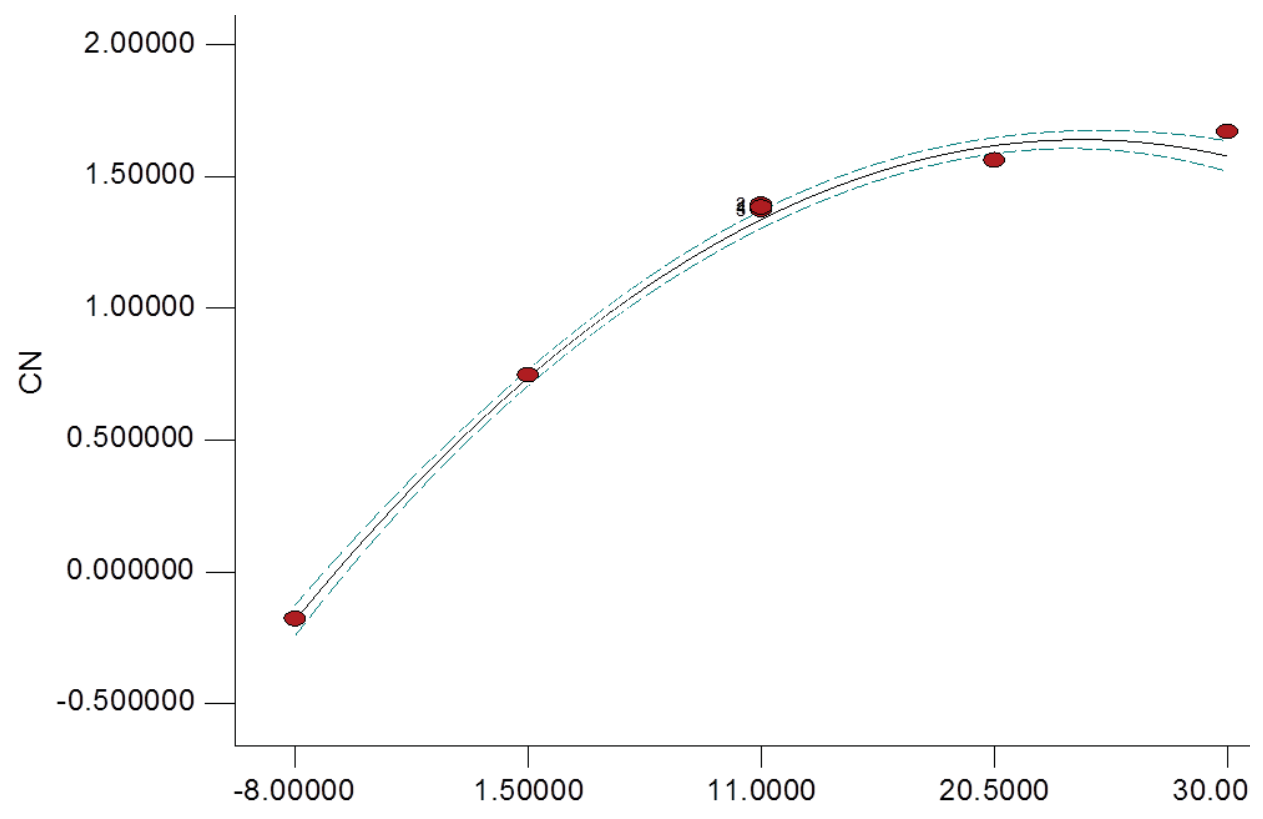

A: aoa (deg)

Figure 8. Normal force as a function of angle of attack showing confidence bands, GL-10 configuration without engines and zero surface deflections.

Table 7. ANOVA for Dwell Test

\begin{tabular}{ccccccc} 
Source & $\begin{array}{c}\text { Sum of } \\
\text { Squares }\end{array}$ & df & MS & F & p-value & significant \\
\hline \hline Block & 0.4584 & 1 & 0.4584 & & & \\
Model & 11.3387 & 9 & 1.2599 & 250.57 & $2.6 E-19$ & $\mathrm{x}$ \\
A-aoa & 9.6987 & 1 & 9.6987 & 1928.97 & $3.8 E-22$ & $x$ \\
$B$-beta & 0.0001 & 1 & 0.0001 & 0.02 & $8.9 E-01$ & \\
C-dwell time & 0.0000 & 1 & 0.0000 & 0.00 & $9.9 E-01$ & \\
$A B$ & 0.0002 & 1 & 0.0002 & 0.04 & $8.4 E-01$ & \\
$A C$ & 0.0001 & 1 & 0.0001 & 0.02 & $8.8 E-01$ & \\
$B C$ & 0.0004 & 1 & 0.0004 & 0.08 & $7.8 E-01$ & \\
$A \wedge 2$ & 1.0903 & 1 & 1.0903 & 216.86 & $1.5 E-12$ & $x$ \\
$B \wedge 2$ & 0.0034 & 1 & 0.0034 & 0.68 & $4.2 E-01$ & \\
$C \wedge 2$ & 0.0000 & 1 & 0.0000 & 0.00 & $9.8 E-01$ & \\
Residual & 0.1056 & 21 & 0.0050 & & & \\
Lack of Fit & 0.1054 & 19 & 0.0055 & 50.53 & $2.0 E-01$ & \\
Pure Error & 0.0002 & 2 & 0.0001 & & & \\
Cor Total & 11.9027 & 31 & & & & \\
& & & & & &
\end{tabular}




\section{Aerodynamic Behavior and Hysteresis Check}

A series of conventional sweeps were performed to determine if the aerodynamic models needed to reflect static hysteresis. Sweeps of increasing and decreasing angle of attack and sideslip revealed a level of static hysteresis that was not sufficiently large in any of the coefficients to require special modeling for that characteristic. An example of the limited hysteresis found is shown in Fig. 9 where relatively small differences are seen in normal force coefficient values for angles of attack above $5^{\circ}$. For this study, a slightly larger measurement error was deemed acceptable rather than increase the complexity of the models and experiment design to model the small hysteresis effects.

These data confirmed the aircraft designer's expectation that a stall break would occur in the $10^{\circ}$ to $15^{\circ}$ angle of attack range and lift would be well maintained after stall $1^{10}$. Since aerodynamic coefficients are very strong functions of angle of attack and sideslip, the data supported a separate low range test below $10^{\circ}$ alpha for the cruise mode.

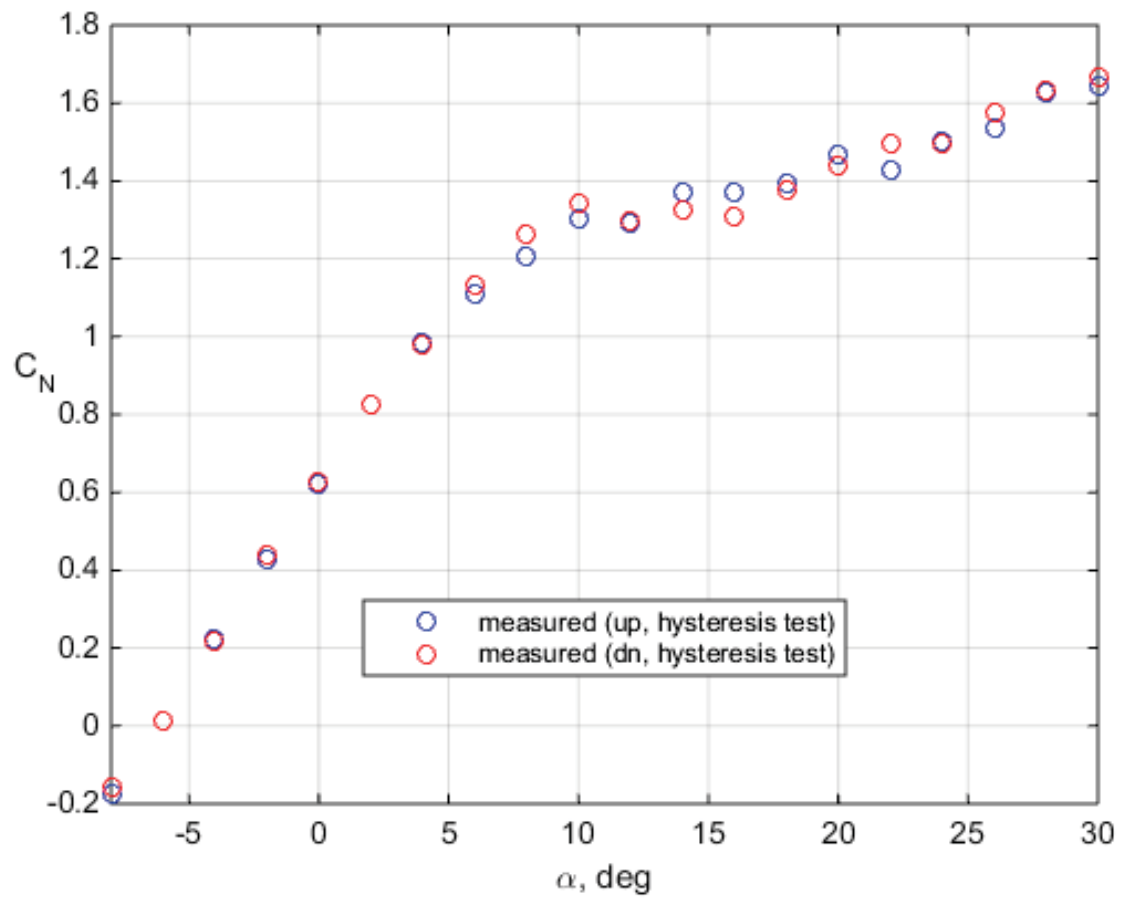

Figure 9. Normal force measurements to assess hysteresis.

\section{Experiments for Each Flight Mode}

A common characteristic of the designs for each flight mode is that a combination of a nested FCD and I-optimal designs were used. Retaining the nested FCD ensured that each corner and edge of the factor ranges were tested. This feature is especially important in aerospace applications where the factors often represent aerodynamic surfaces with nonlinear response characteristics. Developing adequate flight control designs with adequate margins requires full characterization of the control power available to an aircraft.

\section{Cruise \& Loiter Modes}

The final design for the 14-factor Cruise mode required 385 data points (or "runs" in DOE terminology). Final experiment design test points are shown for the Cruise mode in Fig. 10. This is a representative set of test points shown in terms angle of attack and sideslip. The limited ranges for angle of attack $\left(-5^{\circ}\right.$ to $\left.10^{\circ}\right)$ and sideslip $\left(-5^{\circ}\right.$ to $\left.5^{\circ}\right)$ reflect the expectation of a conventional flight envelope in this mode. Similar graphics were obtained for any combination of factors considered. The four colors black, red, green, and blue, correspond to the test blocks 1 to 4, respectively. Blocks 1 and 2 define a nested FCD. Block 1 (black) is the outer FCD and block 2 (red) is the inner FCD. Block 3 (green) provided the optimized test points that minimize prediction error (I-optimal). Block 4 (blue) provided validation points and additional data if needed. 
Loiter mode was tested using the same design as developed for the Cruise mode, except angle-of-attack range was shifted to cover alpha from $5^{\circ}$ to $20^{\circ}$. Sideslip range remained the same $\pm 5^{\circ}$. In this case, because the stall break was included some cubic behavior was expected with respect to angle of attack, in particular for the normal force coefficient.

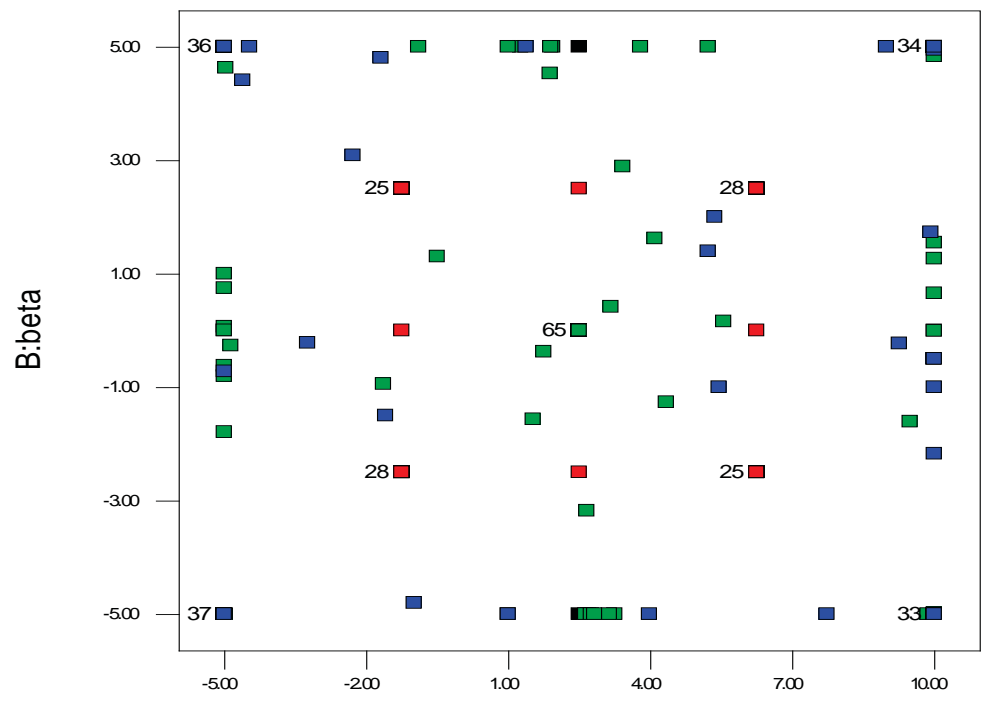

A:aoa

Figure 10. Nested face-centered design with optimized test points for Cruise mode.

\section{Transition Modes: TransLo \& TransHi}

Transition mode was primarily defined by wing tilt. When the wing is stowed in normal flight, then the mode of flight is either Cruise or Loiter. When wing tilt is at $90^{\circ}$, the mode of flight is Hover. Consequently, Transition mode covers an extremely large range of angle of attack for the wing. Breaking this mode into separate tests based on angleof-attack ranges that reflect the aerodynamic behavior is the ideal case, however actuator constraints only allowed a maximum of $45^{\circ}$ angle change. The solution chosen was to break the Transition mode into 2 tests: TransLo covering wing tilt from $0^{\circ}$ to $45^{\circ}$ and TransHi covering wing tilt from $45^{\circ}$ to $90^{\circ}$. The actuator constraint also prevented a design that would have the high and low wing-tilt ranges overlap.

The final design for the 23-factor TransLo mode required 850 runs (test points). Final design test points are shown for the TransLo mode in Fig. 11. The figure shows the nested FCD augmented with I-optimal test points. Angle of attack and sideslip are the two factors selected for this plot as representative of test points for all the modes and factors. The limited ranges for angle of attack $\left(0^{\circ}\right.$ to $\left.15^{\circ}\right)$ and sideslip $\left(-5^{\circ}\right.$ to $\left.5^{\circ}\right)$ reflect the expected flight envelope in this mode. Similar graphics were obtained for any combination of factors considered. The four colors black, red, green, and blue, correspond to the four design test blocks 1 to 4 , respectively. Blocks 1 and 2 define a nested FCD and blocks 3 and 4 are the optimized test points chosen to minimize prediction error. A similar design with 850 runs was developed for the TransHi test except the range for wing tilt changed to cover $45^{\circ}$ to $90^{\circ}$. Tail tilt was also adjusted differently between the two transition modes, since it was expected to roughly trend up and down with the wing tilt factor. A different actuator for tail tilt allowed an overlap in range between the high and low transition tests. In TransLo the tail tilt range covered $-8^{\circ}$ to $50^{\circ}$ and in TransHi the range it was $45^{\circ}$ to $98^{\circ}$.

American Institute of Aeronautics and Astronautics 


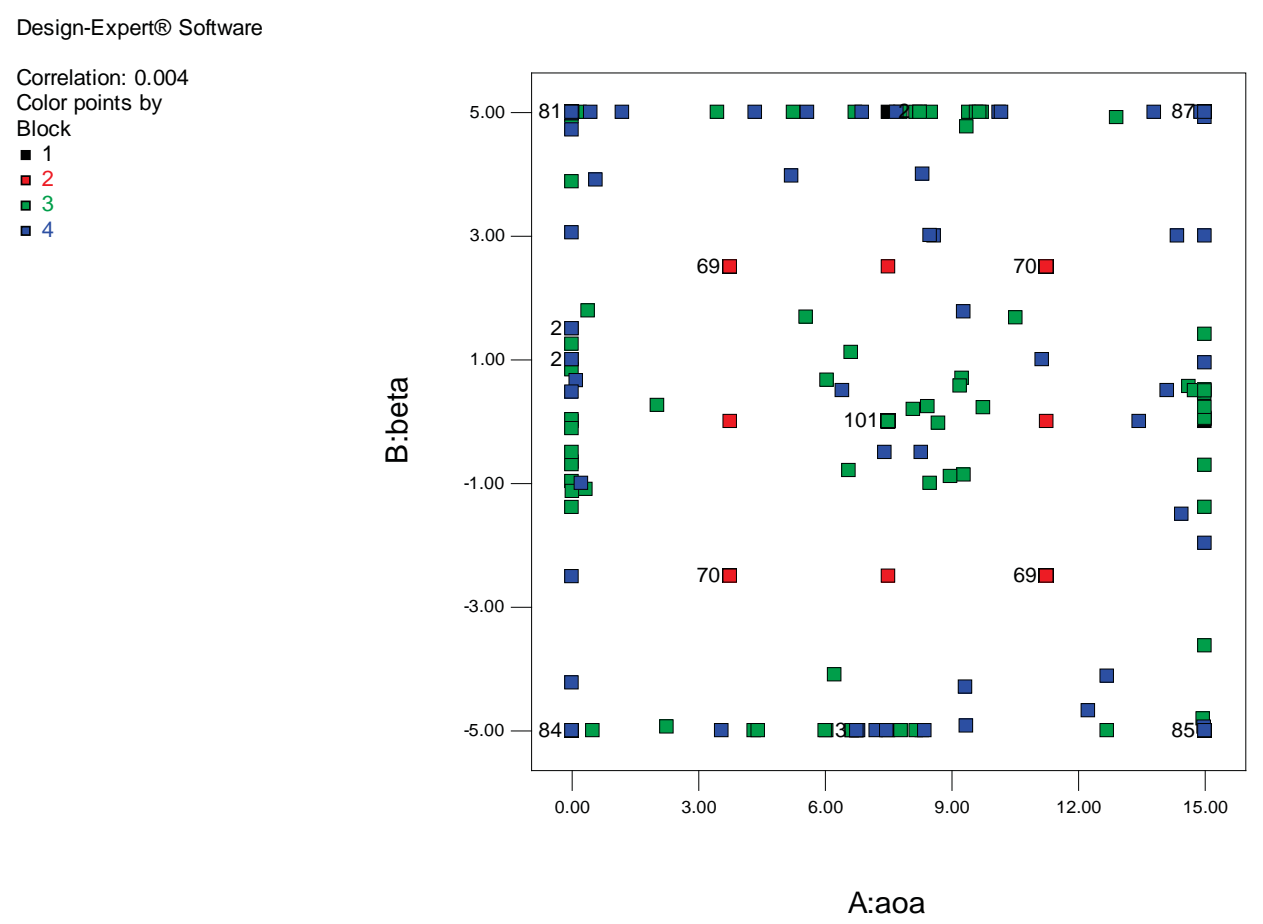

Figure 11. Nested FCD blended with optimized test points for TransLo mode.

\section{Hover Mode}

Hover mode is characterized by both the wing and tail in a vertical configuration. The Hover mode test was designed to test flight conditions similar to take-off conditions without any wind or forward speed. Consequently the wind was off during this test and all the forces and moments were generated by the aircraft propulsion system. In this case with 19 factors, 622 runs were required to satisfy the design metrics described previously. Design patterns showing final design test points for each factor pair are very similar to that shown for the TransLo mode in Fig. 11 or the Cruise mode in Fig. 10. The Hover mode test was designed using the same combination of a nested FCD blended with optimized test points chosen to minimize prediction error.

\section{Experimental Results}

The data collected were analyzed using stepwise regression, a variation of ordinary least-squares regression, where regressors are added or removed in a sequential manner based on significance testing. Significance levels were set very high ( $\alpha=1 \%)$ to ensure only the most significant features of the aerodynamic models were retained. Cubic models were also evaluated to capture any important $3^{\text {rd }}$ order terms. Data analysis and significance testing were accomplished with the aid of Design Expert ${ }^{\mathrm{TM}}$, a commercially available program. Final regressors were evaluated primarily through ANOVA, however engineering judgment was required to remove some terms that were still statistically significant. Although these terms were statistically significant they represented relatively small aerodynamic effects and were not consistent with symmetric aircraft aerodynamics. These terms may have arose due to anomalous sources such as tunnel-flow asymmetries, set-point errors, or measurement noise.

The final models in this study were not forced to have perfect symmetry. For example, no requirement was made that the right outboard flap have exactly the same control effectiveness as the left outboard flap. These types of differences reflect the nature of experimental testing. However, additional treatment of the models forcing perfect symmetry was done later for an aircraft simulation developed primarily for stability and control analysis and design. Delaying the imposition of symmetry initially allows complete discovery of all model characteristics as tested including any unexpected interactions that may be present. The measurements reflect responses of the system under test as well as the quality of the measurement tools, procedures, and facility itself. This approach allows any anomalies to be observed during testing and the use of engineering judgment to make corrections if and where needed.

American Institute of Aeronautics and Astronautics 
To moderate the length of this paper, only sample models from each mode will be shown graphically and details of ANOVA and model terms will be limited to one example in the TransLo region of the Transition mode.

\section{A. Test Results for Cruise Mode}

Analysis of Cruise mode data suggested that a quadratic model for normal force is adequate. Multiple correlation coefficients, $\left(R^{2}\right.$, adj- $R^{2}$, prediction $\left.R^{2}\right)$ were estimated to be $98 \%$ or higher. $R^{2}$ indicates how well the variation in the model explains the total variation in the data; its values range between 0 and 1 . Adj- $R^{2}$ adjusts for the number of parameters in the model, penalizing over-parameterization, and prediction $R^{2}$ provides a metric for the models ability to predict responses. Similar values were found for all six aerodynamic coefficients. These models cover all main effects, 2-factor interactions, and a variety of quadratic behaviors, such as normal force with angle of attack. A surface plot showing the estimated normal force model as a function of angle of attack and tail-tilt (Ttilt) is shown in Fig. 12. Measured values are presented on the chart as red dots if they are above the predicted surface and pink dots if they are below. Limited measured values are shown for clarity. Five measured values are shown at the center of the range for each independent factor. The remaining factors were set to their mid-range values to create this surface. The factor values are shown in the legend.
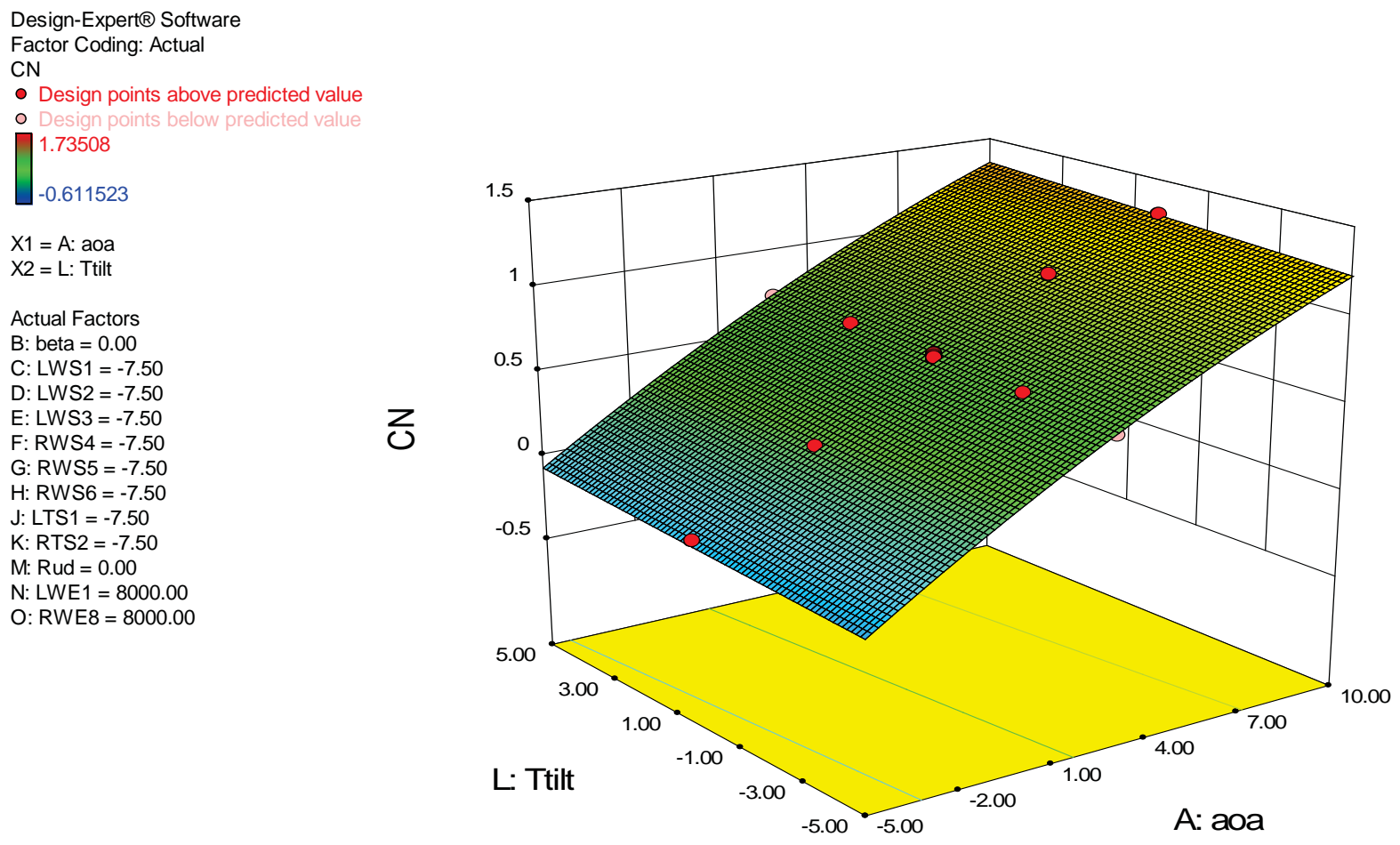

Figure 12. Normal force as a function of angle of attack and tail-tilt in Cruise Mode.

\section{B. Test Results for Loiter Mode}

Analysis of Loiter mode data resulted in aerodynamic models that included main effects, 2-factor interactions, and quadratic behaviors. Cubic terms were also included in all coefficients except side force and rolling moment. Normal force presented a cubic behavior with angle of attack, as shown in Fig. 13. The angle of attack region considered for Loiter mode captured the stall break and the very flat behavior beyond stall. Researchers hypothesize that the motor pylons act as large vortilons that enhance lift at high angles of attack. This is consistent with the result that adding vortilons to the wing had little effect. Pitching moment analysis also found a mixed cubic term, $\mathrm{aoa}^{2 *} \mathrm{Tt}$ tilt $\left(\mathrm{A}^{2} \mathrm{~L}\right)$, to be significant. Multiple correlation coefficients, $\left(R^{2}\right.$, adj- $R^{2}$, prediction $\left.R^{2}\right)$ were estimated to be $95 \%$ or higher for all coefficients in Loiter mode. A surface plot showing the estimated pitching moment model as a function of angle of attack and tail-tilt (Ttilt) is shown in Fig. 14. Measured values are again presented as red dots if they are above the model-predicted surface and pink dots if they are below. Remaining factors were set to their mid-range values to create this surface. Factor values are shown in the legend.

American Institute of Aeronautics and Astronautics 
Design-Expert ${ }^{\circledR}$ Software

Factor Coding: Actual

- Design points above predicted value

- Design points below predicted value

1.92553

0.463301

$\mathrm{X} 1=\mathrm{A}:$ aoa

X2 = L: Ttilt

Actual Factors

B: beta $=0.00$

C: LWS1 $=-7.50$

D: $L W S 2=-7.50$

E: LWS3 $=-7.50$

$F:$ RWS4 $=-7.50$

G: RWS5 = -7.50

$\mathrm{H}:$ RWS6 $=-7.50$

$\mathrm{J}:$ LTS1 $=-7.50$

$\mathrm{K}: \mathrm{RTS} 2=-7.50$

M: Rud $=0.00$

$\mathrm{N}:$ LWE1 $=8000.00$

O: RWE8 $=8000.00$

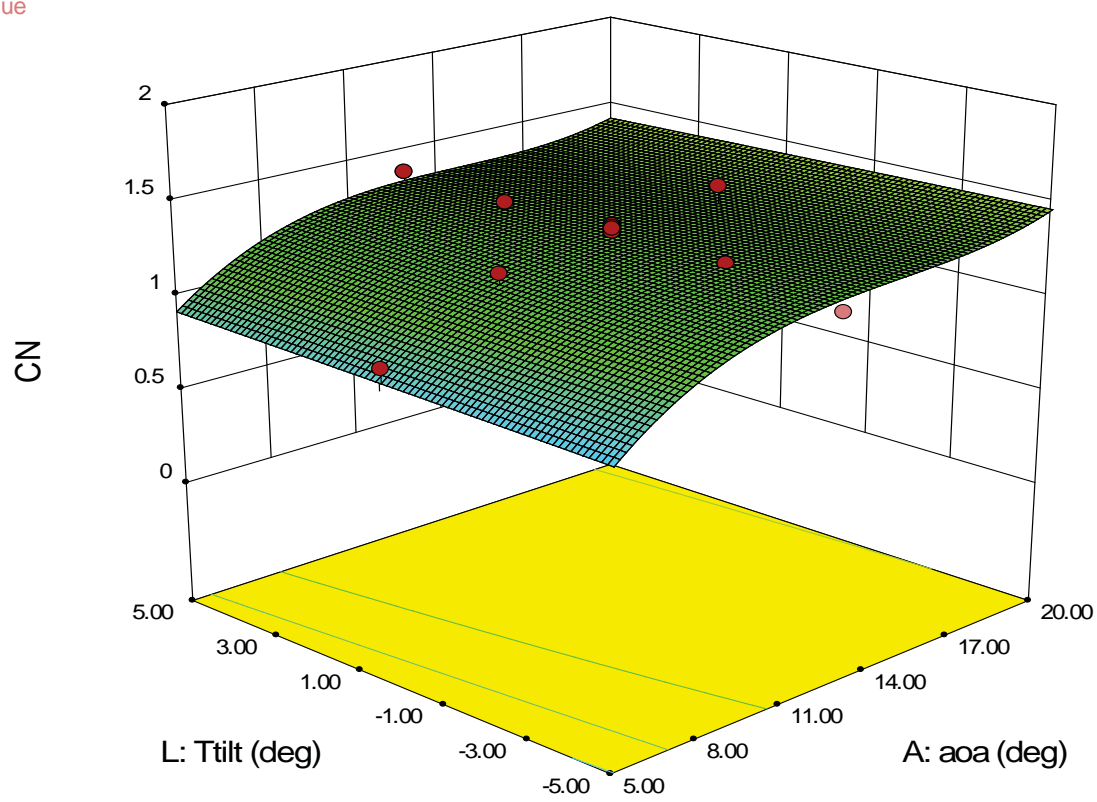

Figure 13. Normal force as a function of angle of attack and tail-tilt in Loiter Mode.

Design-Expert $₫$ Software

Factor Coding: Actual

$\mathrm{Cm}$

- Design points above predicted value

- Design points below predicted value

0.706311

$-0.653082$

$\mathrm{X} 1=\mathrm{A}:$ aoa

X2 = L: Ttilt

Actual Factors

B: beta $=0.00$

C: LWS1 $=-7.50$

D: $\operatorname{LWS} 2=-7.50$

E: LWS3 $=-7.50$

F: RWS4 $=-7.50$

G: RWS5 $=-7.50$

$\mathrm{H}:$ RWS6 $=-7.50$

$\mathrm{J}:$ LTS1 $=-7.50$

$\mathrm{K}: \mathrm{RTS} 2=-7.50$

$\mathrm{M}:$ Rud $=0.00$

$\mathrm{N}:$ LWE1 $=8000.00$

O: RWE8 $=8000.00$

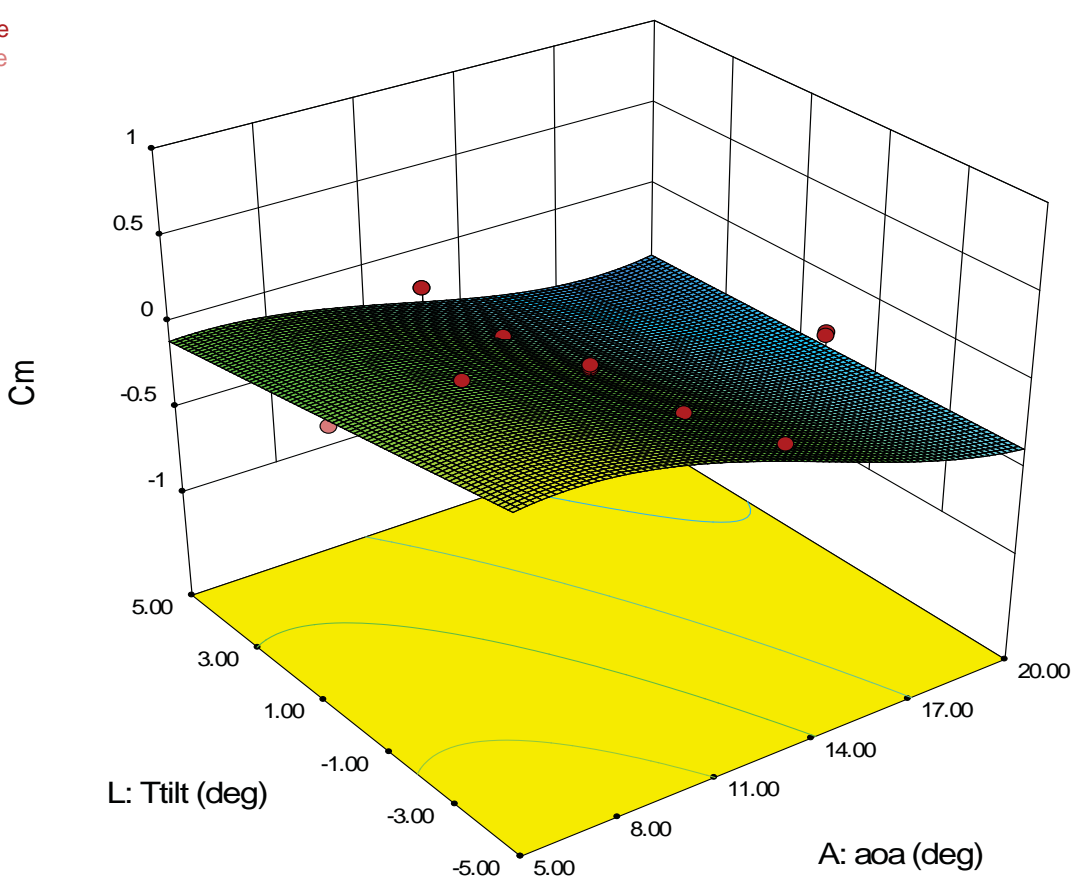

Figure 11-14. Pitching moment as a function of angle of attack and tail-tilt in Loiter Mode.

American Institute of Aeronautics and Astronautics 


\section{Test Results for Transition Mode}

Transition mode presented the most challenging modeling problem. These models, including the various interactions, obtained through the DOE/RSM process would have been extremely difficult to detect and model using conventional OFAT methods. In this case all 23 factors were active and the largest ranges for wing tilt and tail tilt were in effect. Although aircraft angle of attack remained in a relatively modest range from $0^{\circ}$ to $15^{\circ}$, the wing tilt and tail tilt were varied over a full $90^{\circ}$ range which resulted in extreme local angles of attack on the wing and tail. Because of an actuator limitation the wing-tilt angle could only be varied through a range of $45^{\circ}$ in one setup, consequently the transition region was broken into two ranges: (1) wing tilt covering a low range (TransLo) of $0^{\circ}$ to $45^{\circ}$ and (2) a high range (TransHi) of $45^{\circ}$ to $90^{\circ}$. Resources did not allow testing at a series of lower speeds for this study, however this factor would normally be considered given the range of flight conditions in Transition mode.

\section{TransLo Region}

Analysis of Transition mode data, in the TransLo region, resulted in coefficient models that required some cubic and third-order mixed terms for all coefficients except side force. A quadratic model for side force was found to be adequate, reflecting the small range of sideslip tested. For normal force the model covers all main effects (Table 8a), 2 -factor interactions, and is predominately quadratic with one cubic term in wing-tilt and three third-order mixed terms in angle of attack, wing tilt, and tail tilt $\left(\mathrm{A}^{2} \mathrm{~L}, \mathrm{AL}^{2}, \mathrm{LM}^{2}\right)$. Higher order factors are shown in Table 8b. The tables provide values for standard ANOVA terms: Sum of Squares, degree-of-freedom (df), mean square (MS), F-statistic (F), and probability of the term being due to noise (p-value). The last column highlights significant terms, where pvalues were less than $1 \%$.

Table 8a. ANOVA for TransLo CN Main Effects

\begin{tabular}{|c|c|c|c|c|c|c|}
\hline Source & $\begin{array}{l}\text { Sum of } \\
\text { Squares }\end{array}$ & $\mathrm{df}$ & MS & $\mathrm{F}$ & p-value & significant \\
\hline Block & 4.38 & 3 & 1.46 & & & \\
\hline Model & 370.59 & 53 & 6.99 & 690.51 & $<0.0001$ & $x$ \\
\hline A- $а$ oо & 2.59 & 1 & 2.59 & 255.81 & $<0.0001$ & $x$ \\
\hline$C-L W S 1$ & 1.33 & 1 & 1.33 & 131.09 & $<0.0001$ & $x$ \\
\hline$D-L W S 2$ & 2.15 & 1 & 2.15 & 212.68 & $<0.0001$ & $x$ \\
\hline$E-L W S 3$ & 2.28 & 1 & 2.28 & 225.01 & $<0.0001$ & $x$ \\
\hline F-RWS4 & 1.85 & 1 & 1.85 & 182.49 & $<0.0001$ & $x$ \\
\hline G-RWS5 & 1.74 & 1 & 1.74 & 171.75 & $<0.0001$ & $x$ \\
\hline H-RWS6 & 1.34 & 1 & 1.34 & 131.98 & $<0.0001$ & $x$ \\
\hline$J-L T S 1$ & 0.50 & 1 & 0.50 & 49.42 & $<0.0001$ & $x$ \\
\hline$K-R T S 2$ & 0.57 & 1 & 0.57 & 56.24 & $<0.0001$ & $x$ \\
\hline$L$-Wtilt & 1.66 & 1 & 1.66 & 163.86 & $<0.0001$ & $x$ \\
\hline M-Ttilt & 45.91 & 1 & 45.91 & 4533.83 & $<0.0001$ & $x$ \\
\hline$O-L W E 1$ & 3.42 & 1 & 3.42 & 338.10 & $<0.0001$ & $x$ \\
\hline$P-L W E 2$ & 4.42 & 1 & 4.42 & 436.03 & $<0.0001$ & $x$ \\
\hline Q-LWE3 & 7.55 & 1 & 7.55 & 745.13 & $<0.0001$ & $x$ \\
\hline$R-L W E 4$ & 5.49 & 1 & 5.49 & 541.78 & $<0.0001$ & $x$ \\
\hline S-RWE5 & 6.02 & 1 & 6.02 & 594.17 & $<0.0001$ & $x$ \\
\hline T-RWE6 & 6.96 & 1 & 6.96 & 687.48 & $<0.0001$ & $x$ \\
\hline U-RWE7 & 4.80 & 1 & 4.80 & 473.66 & $<0.0001$ & $x$ \\
\hline$V-R W E 8$ & 3.72 & 1 & 3.72 & 367.07 & $<0.0001$ & $x$ \\
\hline W-LTE1 & 4.45 & 1 & 4.45 & 439.02 & $<0.0001$ & $x$ \\
\hline$X$-RTE2 & 4.05 & 1 & 4.05 & 400.42 & $<0.0001$ & $x$ \\
\hline
\end{tabular}


Table 8b. ANOVA for TransLo CN Higer-Order Terms

\begin{tabular}{|c|c|c|c|c|c|c|}
\hline Source & $\begin{array}{l}\text { Sum of } \\
\text { Squares }\end{array}$ & $\mathrm{df}$ & MS & $\mathrm{F}$ & p-value & significant \\
\hline Block & 4.38 & 3 & 1.46 & & & $x$ \\
\hline Model & 370.59 & 53 & 6.99 & 690.51 & $<0.0001$ & $x$ \\
\hline$A L$ & 14.90 & 1 & 14.90 & 1471.50 & $<0.0001$ & $\bar{x}$ \\
\hline$A M$ & 2.36 & 1 & 2.36 & 233.21 & $<0.0001$ & $x$ \\
\hline$C L$ & 0.31 & 1 & 0.31 & 31.07 & $<0.0001$ & $x$ \\
\hline$D L$ & 0.75 & 1 & 0.75 & 73.84 & $<0.0001$ & $x$ \\
\hline$E L$ & 1.21 & 1 & 1.21 & 119.96 & $<0.0001$ & $x$ \\
\hline$F L$ & 1.02 & 1 & 1.02 & 100.39 & $<0.0001$ & $x$ \\
\hline GL & 0.81 & 1 & 0.81 & 79.82 & $<0.0001$ & $x$ \\
\hline$H L$ & 0.62 & 1 & 0.62 & 61.45 & $<0.0001$ & $x$ \\
\hline$J M$ & 0.20 & 1 & 0.20 & 19.79 & $<0.0001$ & $x$ \\
\hline$K M$ & 0.20 & 1 & 0.20 & 19.65 & $<0.0001$ & $x$ \\
\hline$L M$ & 0.027 & 1 & 0.027 & 2.71 & 0.1003 & \\
\hline$L O$ & 1.12 & 1 & 1.12 & 110.70 & $<0.0001$ & $x$ \\
\hline$L P$ & 0.95 & 1 & 0.95 & 94.00 & $<0.0001$ & $x$ \\
\hline$L Q$ & 1.02 & 1 & 1.02 & 101.07 & $<0.0001$ & $x$ \\
\hline$L R$ & 0.54 & 1 & 0.54 & 53.51 & $<0.0001$ & $x$ \\
\hline$L S$ & 0.63 & 1 & 0.63 & 62.63 & $<0.0001$ & $x$ \\
\hline$L T$ & 0.72 & 1 & 0.72 & 71.19 & $<0.0001$ & $x$ \\
\hline$L U$ & 1.06 & 1 & 1.06 & 104.70 & $<0.0001$ & $x$ \\
\hline$L V$ & 0.62 & 1 & 0.62 & 61.56 & $<0.0001$ & $x$ \\
\hline$M W$ & 3.56 & 1 & 3.56 & 351.99 & $<0.0001$ & $x$ \\
\hline$M X$ & 3.05 & 1 & 3.05 & 301.60 & $<0.0001$ & $x$ \\
\hline$A^{\wedge} 2$ & 0.12 & 1 & 0.12 & 12.12 & 0.0005 & $x$ \\
\hline$L \wedge 2$ & 2.51 & 1 & 2.51 & 248.11 & $<0.0001$ & $x$ \\
\hline$M \wedge 2$ & 0.64 & 1 & 0.64 & 62.80 & $<0.0001$ & $x$ \\
\hline$O^{\wedge} 2$ & 0.18 & 1 & 0.18 & 18.01 & $<0.0001$ & $x$ \\
\hline$R^{\wedge} 2$ & 0.28 & 1 & 0.28 & 27.66 & $<0.0001$ & $x$ \\
\hline$S \wedge 2$ & 0.26 & 1 & 0.26 & 25.67 & $<0.0001$ & $x$ \\
\hline$V^{\wedge} 2$ & 0.19 & 1 & 0.19 & 18.40 & $<0.0001$ & $x$ \\
\hline$A^{\wedge} 2 L$ & 0.29 & 1 & 0.29 & 28.91 & $<0.0001$ & $\bar{x}$ \\
\hline$A L^{\wedge} 2$ & 5.12 & 1 & 5.12 & 505.73 & $<0.0001$ & $x$ \\
\hline$L M^{\wedge} 2$ & 0.13 & 1 & 0.13 & 12.71 & 0.0004 & $x$ \\
\hline$L^{\wedge} 3$ & 0.72 & 1 & 0.72 & 71.51 & $<0.0001$ & $x$ \\
\hline Residual & 8.03 & 793 & 0.010 & & & \\
\hline Lack of Fit & 8.01 & 779 & 0.010 & 6.86 & $<0.0001$ & $x$ \\
\hline Pure Error & 0.021 & 14 & $1.498 E-003$ & & & \\
\hline Cor Total & 382.99 & 849 & & & & \\
\hline
\end{tabular}

Two factor interactions shown in Table 8b are significant except for LM which has been retained due to hierarchy requirements ${ }^{7}$. Normally an undesirable result is Lack of Fit being significant. In this case the sources of error (tunnel flow, set-point error, measurement noise, etc.) contributed to some non-physical aircraft asymmetries and in turn non-physical polynomial terms being significant. Terms reflecting the non-physical behaviors were removed from the model and this resulted in significant Lack of Fit. Although not desirable, the result is an aircraft model with slightly poorer parameter estimates. A surface plot showing the estimated normal force model as a function of angle of attack and wing-tilt (Wtilt) is shown in Fig. 15. The figure demonstrates the quadratic and cubic behavior in the normal force coefficient.

American Institute of Aeronautics and Astronautics 
Design-Expert $₫$ Software

Factor Coding: Actual

$\mathrm{CN}$

- Design points above predicted value

- Design points below predicted value

4.22759

$-0.523311$

$\mathrm{X} 1=\mathrm{A}:$ aoa

$\mathrm{X} 2=\mathrm{L}$ : Wtilt

Actual Factors

$\mathrm{B}:$ beta $=0.00$

C: LWS1 $=-7.50$

D: $L W S 2=-7.50$

E: LWS3 $=-7.50$

F: RWS4 $=-7.50$

G: RWS5 $=-7.50$

$\mathrm{H}:$ RWS6 $=-7.50$

$\mathrm{J}:$ LTS1 $=-7.50$

K: RTS2 $=-7.50$

M: Ttilt $=21.00$

$\mathrm{N}:$ Rud $=0.00$

O: LWE1 $=8000.00$

$\mathrm{P}:$ LWE2 $=8000.00$

Q: LWE3 $=8000.00$

R: LWE4 $=8000.00$

S: RWE5 $=8000.00$

T: RWE6 $=8000.00$

$\mathrm{U}: \mathrm{RWE} 7=8000.00$

V: RWE8 $=8000.00$

$\mathrm{W}:$ LTE1 $=8000.00$

$\mathrm{X}: \mathrm{RTE2}=8000.00$

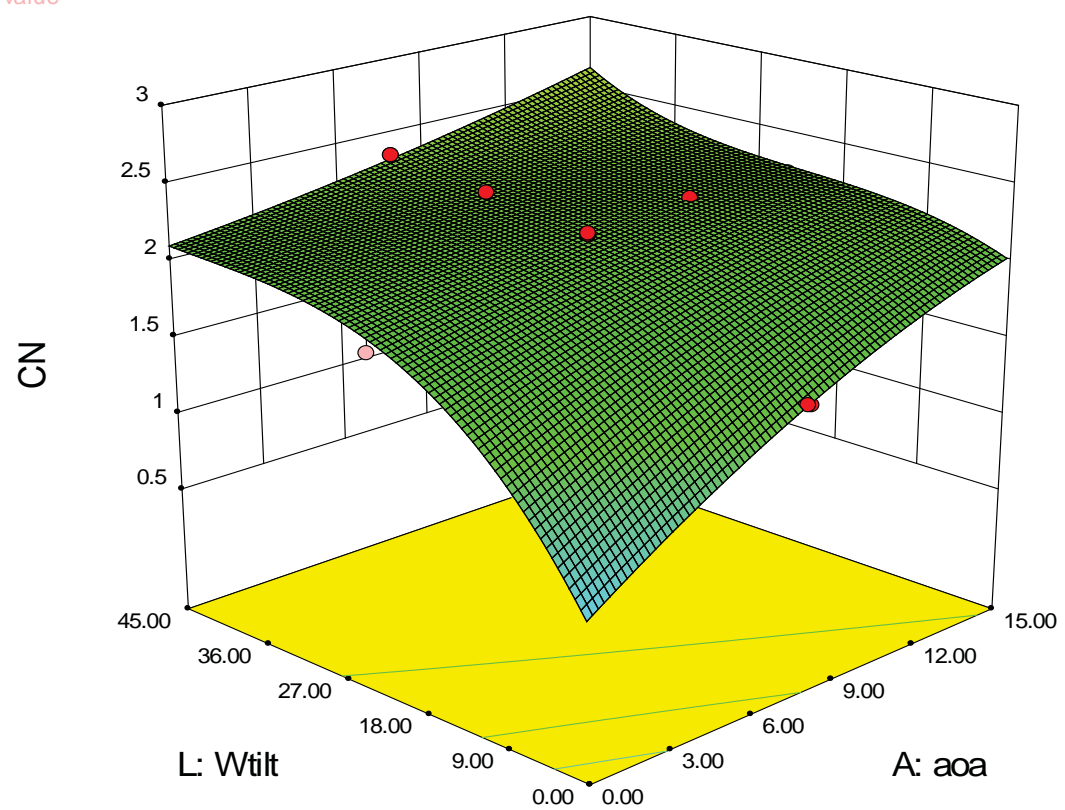

Figure 15. Normal force as a function of angle of attack and wing-tilt in TransLo region.

Design-Expert $\circledast$ Software
CN
Color points by
Block
- 1
2
3
- 4

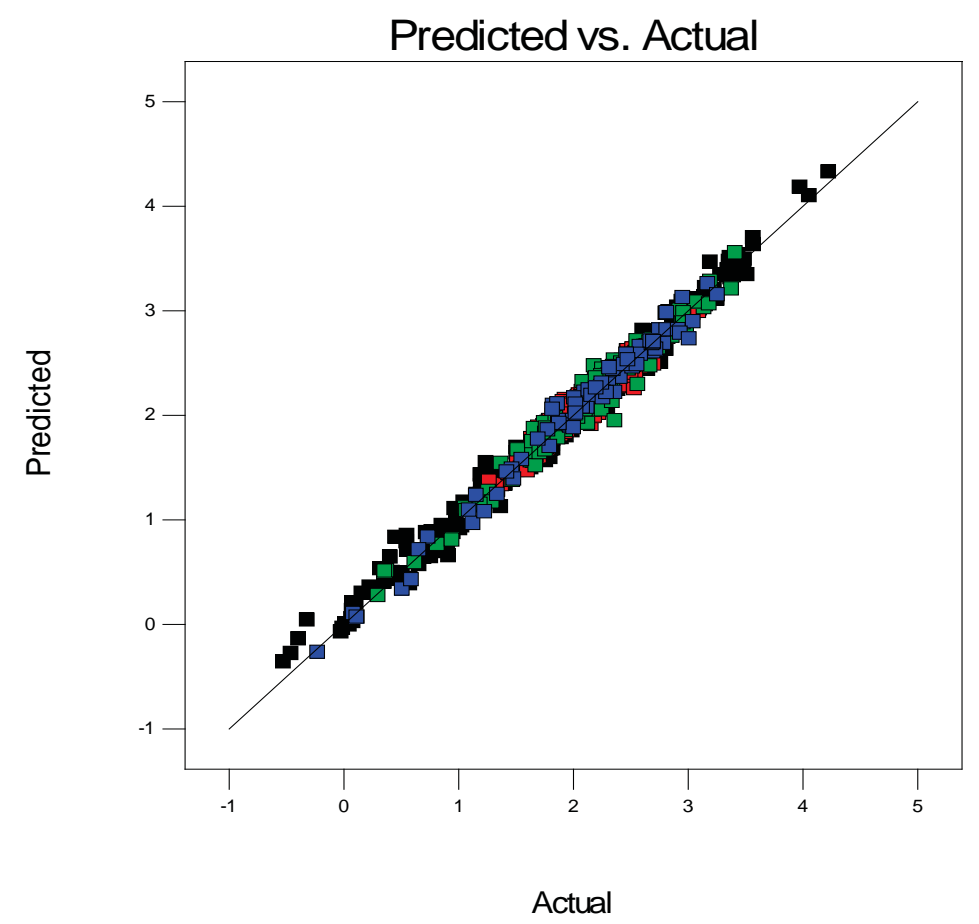

Figure 16. Normal force prediction versus actual measurments for all 4 blocks.

American Institute of Aeronautics and Astronautics 
To demonstrate normal-force model performance, model prediction versus actual measurements are presented in Fig. 16. The figure validates the model by showing good prediction against measurements for all four blocks of data. Block 4 runs, shown in blue, are validation runs and model predictive performance was similar for models estimated using data from blocks 1 to 3 or using all data including block 4. Similar results were obtained for other coefficients.

The pitching moment model presents significant terms in main effects, 2-factor interactions, quadratic terms, one cubic term in wing-tilt, and six 3-factor interaction or mixed cubic terms (AMR, AMS, AMW, AMX, AL ${ }^{2}, \mathrm{LM}^{2}$ ). A surface plot showing the estimated pitching moment model as a function of angle of attack and wing-tilt is shown in Fig. 17. This figure highlights the interaction term $\mathrm{AL}^{2}$.

To highlight the 3-factor interactions (AMW, AMX), Figs. 18a, 18b, and 18c, present 2-dimensional plots of the AM interaction terms for three tail engine (W, X) settings: low (4000 rpm), mid-range (8000 rpm), high (12000 rpm). The tail engines are set together at the same values. Fig. 18a presents the pitching moment versus tail tilt (M: Ttilt) for a low angle of attack $\left(\mathrm{A}\right.$ : aoa $\left.=0^{\circ}\right)$ and a high angle of attack $\left(\mathrm{A}\right.$ : aoa $\left.=15^{\circ}\right)$ with tail engines at mid-range or 8000 $\mathrm{rpm}$. The need for the interaction term AM, a product of angle of attack (A) and tail tilt (M) is demonstrated by virtue of the two graphs not being parallel. Green measured points reflect the mid-range values and center points as one moves from the low to high angle-of-attack values. Figures 18b and 18c highlight the significant change in the interaction characteristic, i.e., the relationship between angle of attack (A) and tail-tilt (M) as the tail engines (W, X) are varied from idle to maximum rpm or from $4000 \mathrm{rpm}$ to $12000 \mathrm{rpm}$. The significant variation in the AM interaction with tail engines required the AMW and AMX terms in the final model.
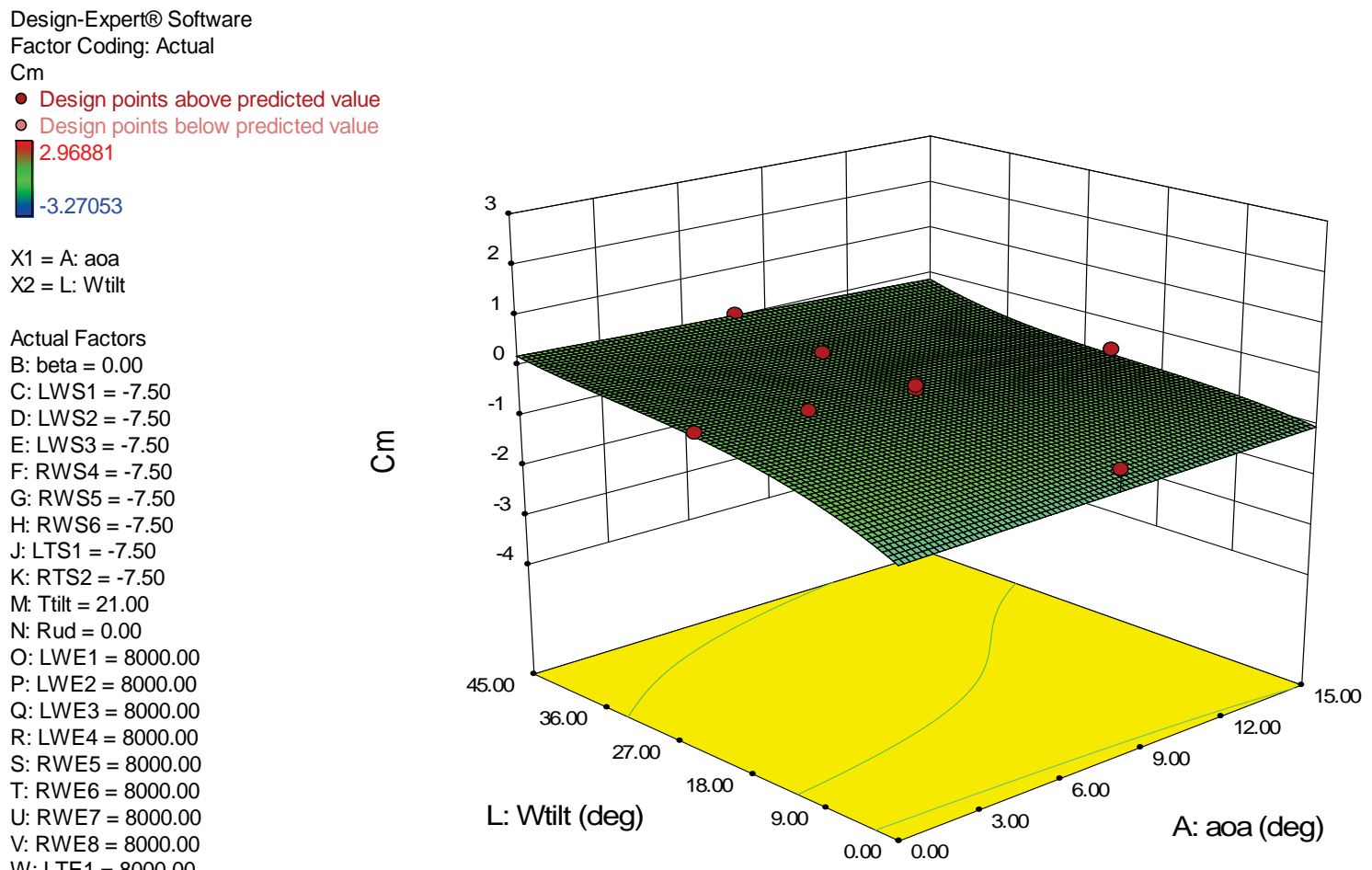

Figure 17. Pitching moment as a function of angle of attack and wing-tilt in TransLo region. 
Design-Expert ${ }^{\circledR}$ Software

Factor Coding: Actual

$\mathrm{Cm}$

- Design Points

$\mathrm{X} 1$ = M: Ttilt

$\mathrm{X} 2$ = A: aoa

Actual Factors

B: beta $=0.00$

C: LWS1 $=-7.50$

D: $L W S 2=-7.50$

E: LWS3 = -7.50

$F:$ RWS4 $=-7.50$

G: RWS5 $=-7.50$

H: RWS6 $=-7.50$

$\mathrm{J}:$ LTS1 $=-7.50$

$\mathrm{K}: \mathrm{RTS} 2=-7.50$

$\mathrm{L}:$ Wtilt $=22.50$

$\mathrm{N}:$ Rud $=0.00$

O: LWE1 $=8000.00$

P: LWE2 $=8000.00$

$\mathrm{Q}:$ LWE3 $=8000.00$

R: LWE4 $=8000.00$

S: RWE5 $=8000.00$

T: RWE6 $=8000.00$

$\mathrm{U}: \mathrm{RWE7}=8000.00$

V: RWE8 $=8000.00$

$\mathrm{W}:$ LTE1 $=8000.00$

$\mathrm{X}:$ RTE2 $=8000.00$

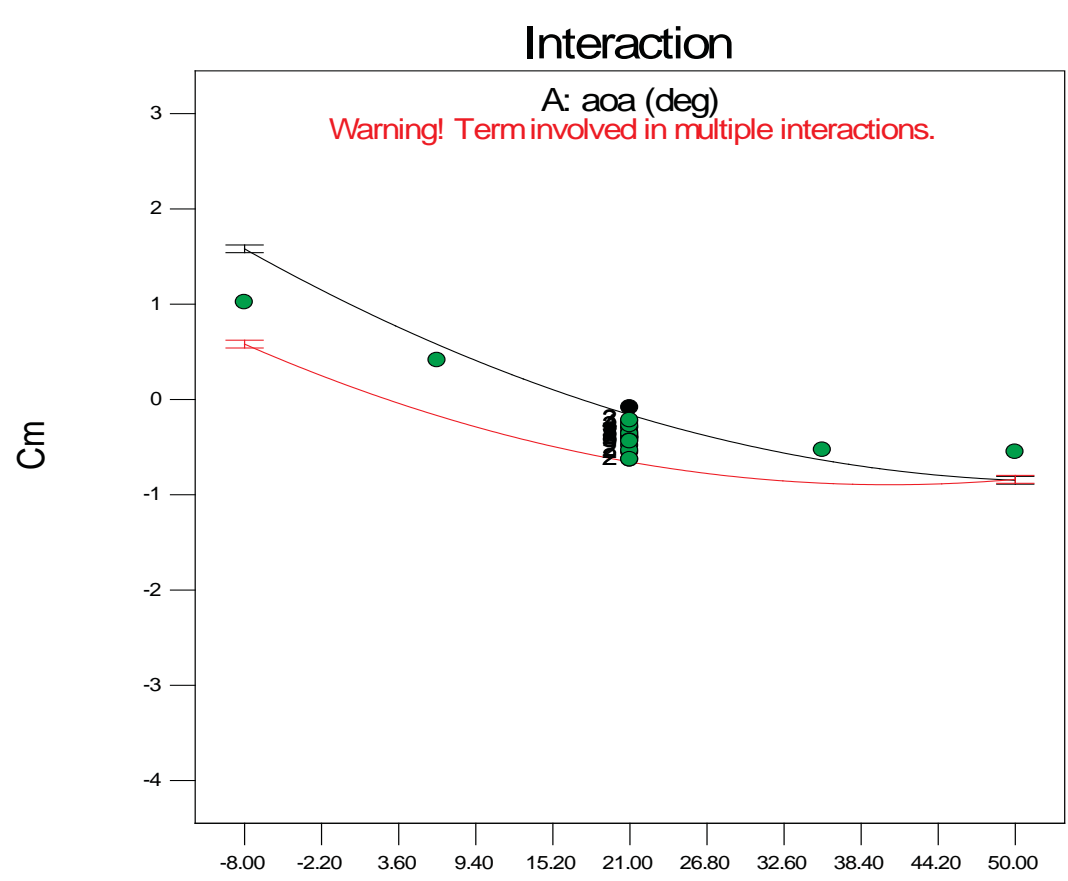

M: Ttilt (deg)

A- 0.00

$\Delta A+15.00$

Figure 18a. $\mathrm{Cm}$ as a function of tail-tilt and angle of attack in TransLo region.

Design-Expert@ Software Factor Coding: Actual $\mathrm{Cm}$

$\mathrm{X} 1$ = M: Ttilt

$\mathrm{X} 2=\mathrm{A}:$ aoa

Actual Factors

B: beta $=0.00$

C: LWS1 $=-7.50$

D: LWS2 $=-7.50$

E: $L W S 3=-7.50$

F: RWS4 $=-7.50$

G: RWS5 $=-7.50$

$\mathrm{H}:$ RWS6 $=-7.50$

$\mathrm{J}:$ LTS1 $=-7.50$

$\mathrm{K}: \mathrm{RTS} 2=-7.50$

$\mathrm{L}: \mathrm{Wtilt}=22.50$

$\mathrm{N}:$ Rud $=0.00$

O: LWE1 $=8000.00$

$P:$ LWE2 $=8000.00$

$\mathrm{Q}:$ LWE3 $=8000.00$

R: LWE4 $=8000.00$

S: RWE5 $=8000.00$

T: RWE6 $=8000.00$

$\mathrm{U}: \mathrm{RWE7}=8000.00$

V: RWE8 $=8000.00$

W: LTE1 $=4000.00$

$X:$ RTE2 $=4000.00$

- A- 0.00

$\Delta A+15.00$

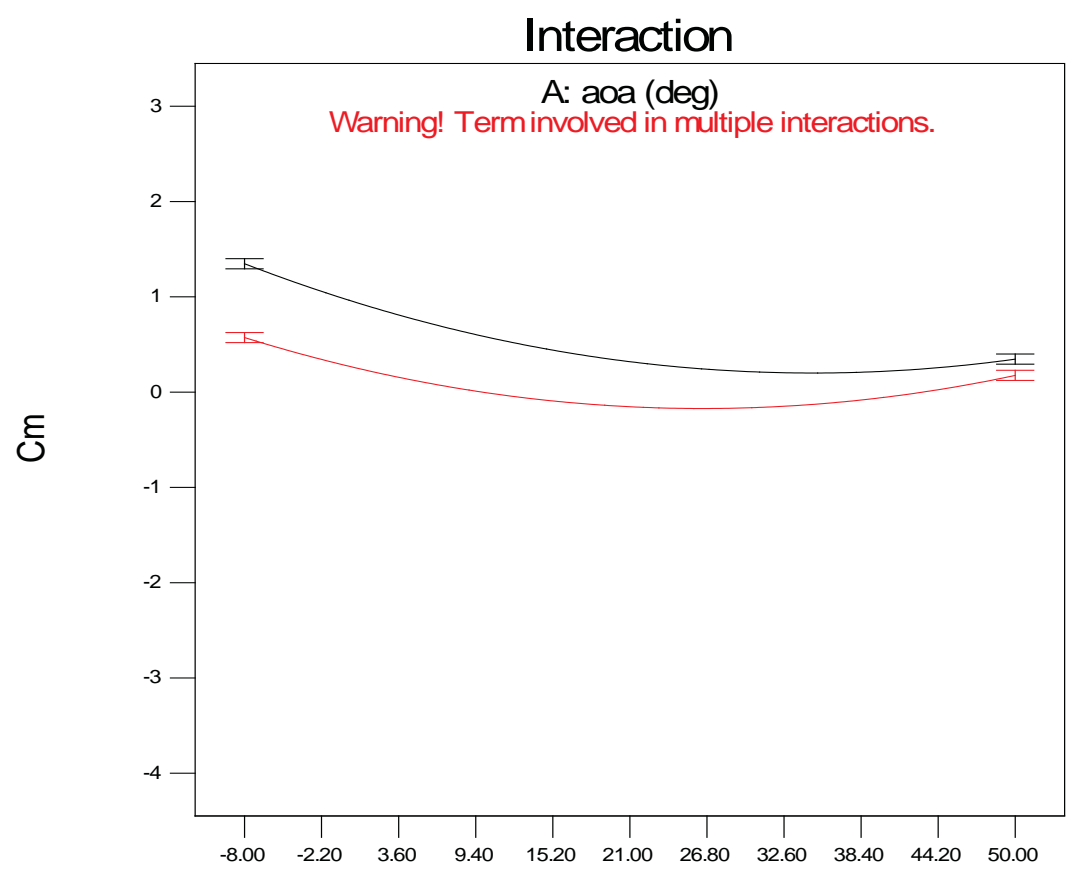

M: Ttilt (deg)

Figure $18 \mathrm{~b}$. $\mathrm{Cm}$ as a function of tail-tilt and angle of attack with tail engines idle. 
Design-Expert ${ }^{\circledR}$ Software

Factor Coding: Actual

$\mathrm{Cm}$

X1 = M: Ttilt

X2 = A: aoa

Actual Factors

B: beta $=0.00$

C: LWS1 $=-7.50$

D: $L W S 2=-7.50$

E: $L W S 3=-7.50$

$F:$ RWS4 $=-7.50$

G: RWS5 $=-7.50$

H: RWS6 $=-7.50$

$\mathrm{J}:$ LTS1 $=-7.50$

$\mathrm{K}: \mathrm{RTS} 2=-7.50$

$\mathrm{L}:$ Wtilt $=22.50$

$\mathrm{N}:$ Rud $=0.00$

O: LWE1 $=8000.00$

$\mathrm{P}:$ LWE2 $=8000.00$

Q: LWE3 $=8000.00$

R: LWE4 $=8000.00$

S: RWE5 $=8000.00$

T: RWE6 $=8000.00$

U: RWE7 $=8000.00$

$\mathrm{V}:$ RWE8 $=8000.00$

W: LTE1 $=12000.00$

$\mathrm{X}:$ RTE2 $=12000.00$

- A- 0.00

$\Delta A+15.00$

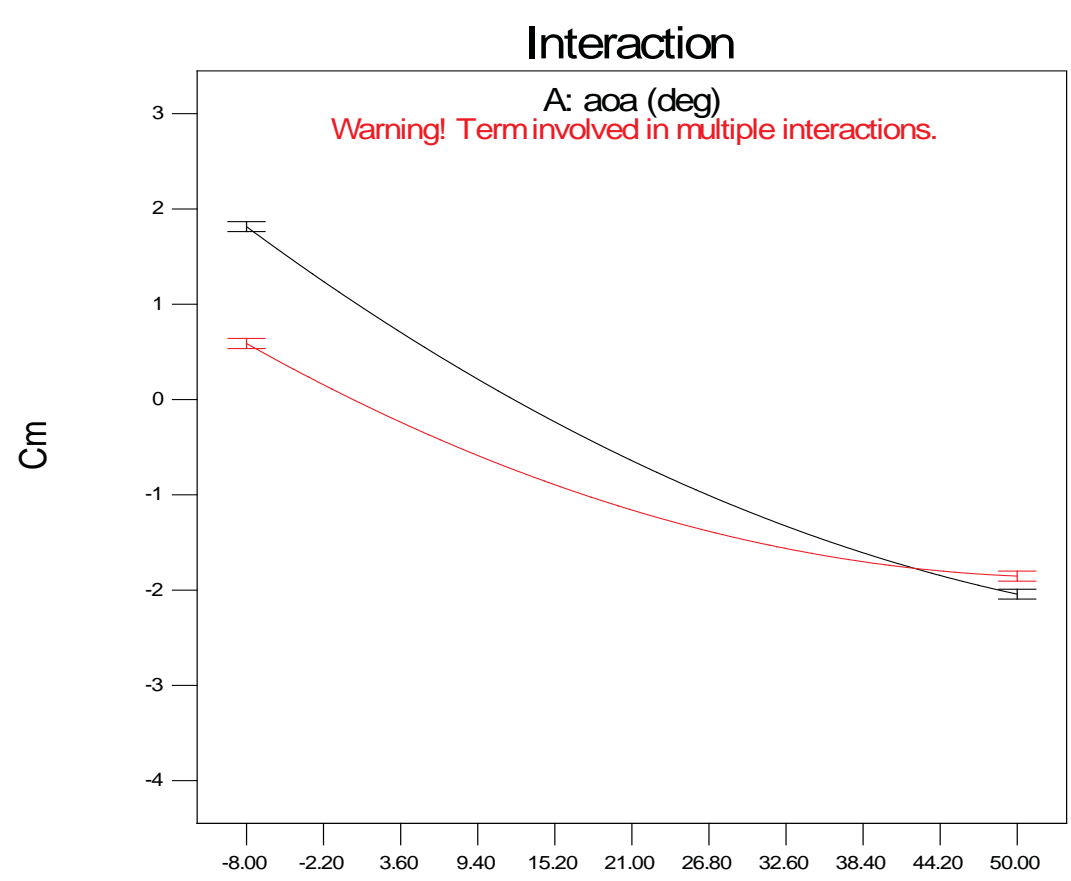

M: Ttilt (deg)

Figure 18c. Cm as a function of tail-tilt and angle of attack with tail engines at maximum rpm.

2. TransHi Region

A graph of normal force in high range transition mode (TransHi) is shown in Fig. 19. In this case a relatively flat surface is presented for normal force against wing tilt and angle of attack with engines and other factors at their midrange values. Analysis for this case found that a cubic term was still statistically significant in wing tilt although only three 3-factor interaction or mixed cubic terms were required. The three terms were ALM, $\mathrm{AL}^{2}$, and $\mathrm{LM}^{2}$. These terms highlight the complex relationships between angle of attack, wing tilt and tail tilt. 


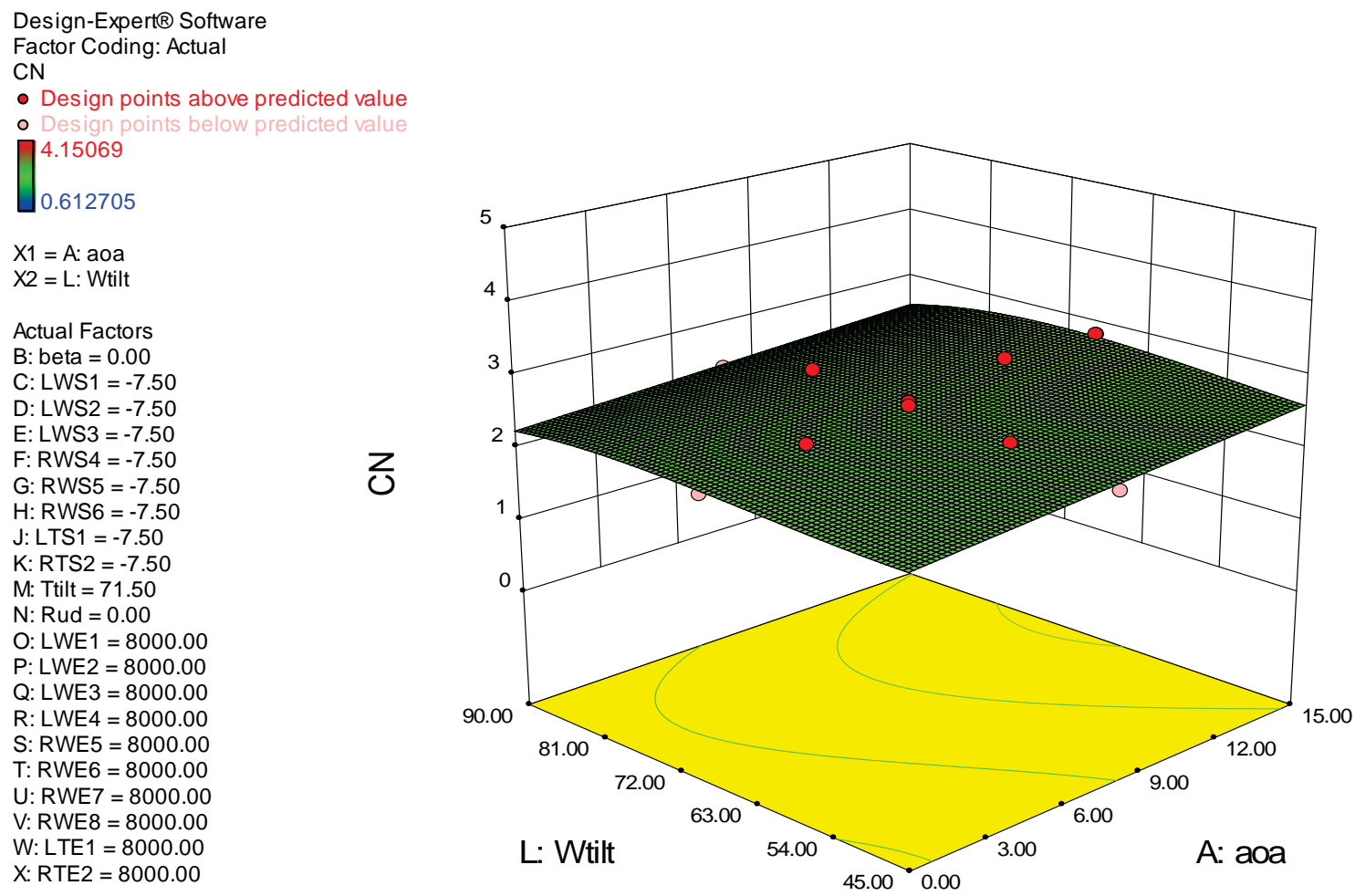

Figure 19. Normal force as a function of angle of attack and wing-tilt in TransHi Mode.

\section{Test results for Hover Mode}

Analysis of Hover mode data produced quadratic models for all coefficients except side force which only required main effects terms. Multiple correlation coefficients, $\left(R^{2}\right.$, adj- $R^{2}$, prediction $\left.R^{2}\right)$ fell into an acceptable range from $85 \%$ to $98 \%$ for all coefficients except side force. Side force correlation coefficients fell to less than $50 \%$. In the Hover mode where wing and tail are vertical and wind tunnel air is off, only relatively small side forces can be generated. The primary side force generator is the rudder but without forward velocity it provides no significant side force. Figure 20 shows the yawing moment as a function of left-wing outboard flap and left-wing outboard engine. The figure highlights the expected interaction between the outboard flap directly behind the outboard engine, showing significantly more yawing moment as the engine rpm is increased. 


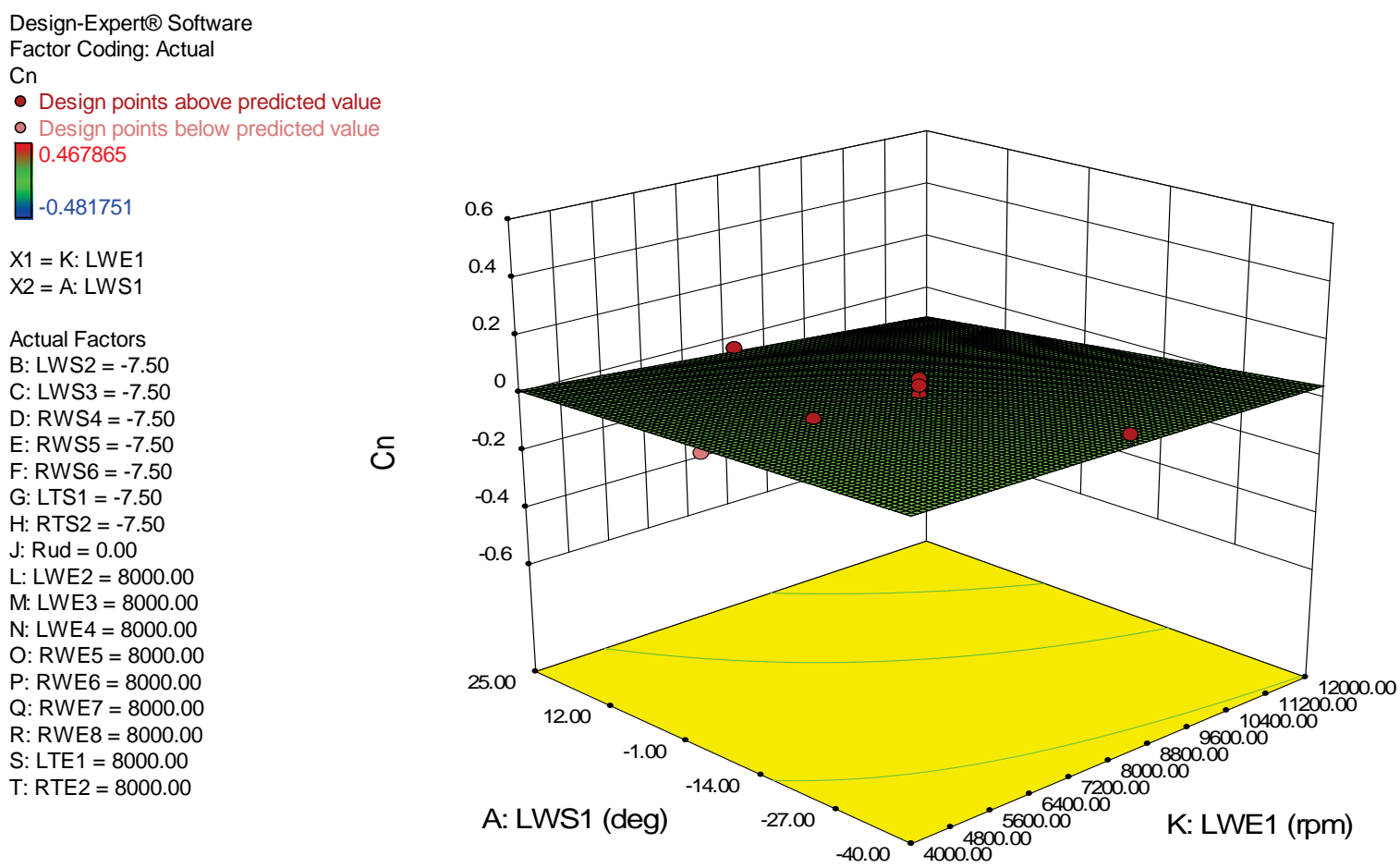

Figure 20. Yawing moment as a function of left-wing flap and left-wing engine in Hover.

\section{Concluding Remarks}

Static tests were conducted at the NASA Langley 12 Foot wind tunnel to model all six aerodynamic coefficients over a large flight envelope for an advanced design concept designated as GL-10. This design is a subscale VTOL aircraft with distributed electric propulsion and tilting wing and tail surfaces. Because of the vehicle complexity conventional test methods were impractical. To overcome this issue a series of tests were designed using DOE/RSM methods. This design process allowed for specification of a general regression model for all force and moment coefficients which included any potential interactions. This was a necessity for testing a new configuration with so many factors and many unknown or unproven effects. ANOVA and significance testing supported model building, showing the relative importance of each model term and its statistical significance. Blocking and randomization provided protection against any lurking noise sources. Benefits of blocking to the hover mode testing were direct as analysis of the residuals in the blocks showed a slight lack of independence. By limiting the testing to short blocks the effect was quantified and minimized. The RSM designs make use of nested FCDs augmented with I-optimal test points to create successful designs. These designs allowed models to be determined in a statistically significant and extremely efficient manner that captured factor interactions that would not likely have been found in a conventionally designed experiment. The series of tests included exploratory tests to define factor ranges, model order, and provide some conventional data for comparison. In addition, a specific DOE test to determine the minimum required data dwell time resulted in reducing the traditional sample duration time by a factor of five. This work supports research at NASA in developing advanced configurations, simulations, and advanced control designs.

Several lessons learned, to date, from testing a complex aircraft using DOE/RSM are worth noting:

- Factor changes should be automated for maximum testing efficiency.

- Factor changes should be individually calibrated and measured to ensure test states are accurately known even if not accurately achieved.

- Ideally, factor commands should be set using feedback control to minimize set point error which, in turn, maximizes effectiveness of the experiment design and accuracy of replication points needed to assess pure error.

American Institute of Aeronautics and Astronautics 
- Using a DOE/RSM modeling approach will capture and combine any asymmetries present in the test setup or the test facility. This has the advantage of providing the experimenter with early warnings of any settings not being achieved or highlighting issues with test apparatus. However, if perfectly symmetric models are needed, such as in simulation of symmetric flaps, then post processing of the models is required.

- Ideally, software that allows processing of data and presentation of results to the researcher during the experiment is preferred over delayed batch processing. Online presentation of data including any conventional measurements adds efficiency and limits the need for re-running tests.

- Once the software and communication process was established to bring the DOE test matrix to the test facility, some efficiency was gained by running conventional tests through the same framework.

- An efficient tare method (wind off removal of gravity forces) should be devised especially for aircraft that undergo configuration changes resulting in c.g. shifts during the tests. One approach is to use DOE/RSM to create a tare model.

- Since the DOE test matrix is independent of calibration curves, unnecessary re-calculation of the test matrix is avoided by implementing calibration curves as part of the test facility system.

- Limited resources prevented additional testing for this study, however a series of different test velocities should be anticipated when testing aircraft with propellers.

\section{Acknowledgments}

The authors extend their appreciation to the NASA Langley Reimar Project, and the Vehicle Systems Safety project for support provided.

\section{References}

${ }^{1}$ Fisher, Ronald A., The Design of Experiments, $9^{\text {th }}$ ed., Macmillan. ISBN 0-02-844690-9, 1971 (1935).

${ }^{2}$ DeLoach, R., “Applications of Modern Experiment Design to Wind Tunnel Testing at NASA Langley Research Center," AIAA 98-0713, 36th AIAA Aerospace Sciences Meeting and Exhibit, Reno, NV, 1998.

${ }^{3}$ Morelli, E. A., and DeLoach, R., - Ground Testing Results Using Modern Experiment Design and Multivariate Orthogonal Functions (Invited), AIAA 2003-0653, 41st AIAA Aerospace Sciences Meeting \& Exhibit, Reno, NV, 2003.

${ }^{4}$ DeLoach, R., Assessment of Response Surface Models Using Independent Confirmation Point Analysis, AIAA 2010-741, 48th AIAA Aerospace Sciences Meeting and Exhibit, Orlando, FL, 2010.

${ }^{5}$ Landman, Drew, Simpson, Jim, Vicroy, Dan D., and Parker, Peter, "Response Surface Methods for Efficient Complex Aircraft Configuration Aerodynamic Characterization,” Journal of Aircraft, Vol. 44, No. 4, July-August 2007.

${ }^{6}$ Landman, Drew, Simpson, Jim, Vicroy, Dan D., and Parker, Peter, "Hybrid Design for Aircraft Wind-Tunnel Testing Using Response Surface Methodologies,” Journal of Aircraft, Vol. 44, No. 4, July-August 2007.

${ }^{7}$ Montgomery, Douglas, C., "Design and Analysis of Experiments,” 8th ed., Wiley, 2013.

${ }^{8}$ Busan, Ronald, Rothhaar, Paul, Croom, Mark, Murphy, Patrick C., Grafton, Sue, O’Neal, Anthony, "Enabling Advanced Wind-Tunnel Research Methods Using the NASA Langley 12-Foot Low Speed Tunnel,” AIAA-2014-3000, AIAA Aviation and Aeronautics Forum and Exposition 2014, Atlanta, GA, 2014.

${ }^{9}$ Fredericks, William J., Moore, Mark D., Busan, Ronald C., "Benefits of Hybrid-Electric Propulsion to Achieve 4x Increase in Cruise Efficiency for a VTOL Aircraft,” AIAA-2013-4342, AIAA Aviation 2013, International Powered Lift Conference, Los Angeles, CA, 2013.

${ }^{10}$ Rothhaar, P., Murphy, P. C., Bacon, B. J., Grauer, J.; NASA Langley Distributed Propulsion VTOL Tilt Wing Aircraft Testing, Modeling, Simulation, Control, and Flight Test Development, AIAA AFM Conference, AIAA Aviation 2014, Atlanta, June 16-20, 2014. AIAA Paper No. 2014-2999, 2014.

${ }^{11}$ Box, G. E. P., and Draper, N. R., (1987), Empirical Model Building and Response Surfaces, John Wiley \& Sons, New York, NY, pp. 424.

${ }^{12}$ Box, G. E. P. and Wilson, K. B., “On the experimental attainment of optimum conditions,” J. Roy. Statist. Soc., Ser. B Metho. Vol. 13, No. 1, pp. 1-45, 1951.

${ }^{13}$ Myers, R. H., and Montgomery, D. C., “Response Surface Methodology,” 2nd ed., Wiley, New York, 2002. 